\title{
Exact Solution Techniques for Two-dimensional Cutting and Packing
}

\author{
Manuel Iori ${ }^{(1)}$, Vinícius L. de Lima ${ }^{(2)}$, Silvano Martello( ${ }^{(3)}$, \\ Flávio K. Miyazawa ${ }^{(2)}$, Michele Monaci ${ }^{(3)}$ \\ (1) DISMI, University of Modena and Reggio Emilia (Italy) \\ (2) Institute of Computing, University of Campinas (Brazil) \\ (3) DEI "Guglielmo Marconi", University of Bologna (Italy)
}

\begin{abstract}
We survey the main formulations and solution methods for two-dimensional orthogonal cutting and packing problems, where both items and bins are rectangles. We focus on exact methods and relaxations for the four main problems from the literature: finding a packing with minimum height, packing the items into the minimum number of bins, finding a packing of maximum value, and determining the existence of a feasible packing.
\end{abstract}

Keywords: Two-dimensional rectangle cutting and packing; Exact methods; Relaxations.

\section{Introduction}

The number of publications on cutting and packing problems has been increasing considerably in recent years. In cutting problems, we are given a set of standardized stock units to be cut into smaller items so as to fulfill a given demand, while in packing problems a set of items has to be packed into one or more containers. These two classes of problems are strongly correlated, as packing an item into a container may be equivalent to cutting an item from a stock unit, and hence the same solution methods are often adopted. In the following, we will denote as bins both the stock units and the containers. In certain applications the unique container is a strip of (theoretically) infinite height.

Cutting and packing problems have been widely studied in the literature both for their theoretical interest and their many practical applications, in which they appear in a number of different variants. Some problems consider one-dimensional items and bins, whereas other problems refer to higher dimensions, like, e.g., the (three-dimensional) container loading problem (see Bortfeldt and Wäscher [28]). Items and bins may have rectangular or general (convex or non-convex) shape. Several practical constraints may also be part of the problems, as cargo stability in container loading problems or the need of producing guillotine patterns in cutting problems. In certain applications, cutting and packing problems are combined with other problems, for example in the integrated lot-sizing and cutting stock problems studied by Melega, Araujo, and Jans [135] or in the routing problems with loading constraints considered by Iori and Martello [99]. Packing problems also appear in many other fields, such as telecommunications (Lodi et al. [117, Martello [126]), newspapers paging (Strecker and Hennig [170]), production (Nesello et al. [144]), scheduling (Kwon and Lee [113]), and maritime logistics (Xu and Lee [181]). Most cutting and packing problems are $\mathcal{N} \mathcal{P}$-hard and very challenging in practice. For this reason, sophisticated solution methods are needed for their solution, motivating the frequent literature updating of this area of research. 


\section{Books and surveys}

Several surveys have been dedicated to solution methods for cutting and packing problems. Specifically, referring to surveys published in the last two decades:

- One-dimensional cutting and packing. Valério de Carvalho [173] reviews linear programming models for one-dimensional bin packing and cutting stock problems. Approximation algorithms for packing problems generally belong to two main categories: (i) on-line algorithms sequentially pack the items in the order encountered on input, without knowledge of items not yet packed; (ii) off-line algorithms have complete information about the item set, and may perform preprocessing, reordering, grouping, etc. before packing. Approximation results (for both on-line and off-line algorithms) have been reviewed by Coffman et al. [54] (Sections 3 and 4, respectively). A recent survey on mathematical models and exact algorithms for one-dimensional packing problems was presented by Delorme, Iori, and Martello [65], who also set up a library, the BPPLIB [67, of computer codes and benchmark instances (see http://or.dei.unibo.it/library/bpplib). The books by Martello and Toth [130 and Kellerer, Pferschy, and Pisinger [109] present a comprehensive treatment on the knapsack problem and its variants but do not consider the corresponding two-dimensional versions;

- Two-dimensional rectangular shape cutting and packing. After the classical 2002 surveys by Lodi, Martello, and Vigo [120] and Lodi, Martello, and Monaci [116], a partial updating was presented by the same authors in 2014 [118. The following results appeared in the last decade. A survey on guillotine packing was produced by Ntene and van Vuuren [145]. Silva, Oliveira, and Wäscher [168] reviewed exact and heuristic algorithms for a particular problem (pallet loading) in which all items are identical, including a thorough analysis of benchmark instances and methodologies adopted in the literature for numerical experiments. Oliveira et al. 146] presented a review of heuristic algorithms for the strip packing problem. Christensen et al. 42 proposed a survey on approximation and on-line algorithms, also including a list of open problems in this area. Recently, Russo et al. [161 presented a survey on relaxations for two-dimensional cutting problems with guillotine constraints and a categorization of the resulting bounds, while Bezerra et al. [26] reviewed models for the two-dimensional level strip packing problem;

- Two-dimensional irregular shape cutting and packing. To the best of our knowledge, the first survey dedicated to the packing of irregular shapes into rectangular containers was presented in the Nineties by Dowsland and Dowsland [69]. More recently, two tutorials by Bennell and Oliveira [22, 23. reviewed the main geometric methodologies and algorithmic approaches for the heuristic solution of these problems. The latest survey on this area, presented by Leao et al. [114 in 2020, provides an extensive review of mathematical models for packing irregular shaped objects both in rectangular and irregular containers;

- Multi-dimensional cutting and packing. In 1990, Dyckhoff [71] proposed a typology of cutting and packing problems (in one, two and three dimensions). About twenty years later, a successful improved typology was proposed by Wäscher, Hau $\beta$ ner, and Schumann [178]. More recently, Bortfeldt and Wäscher [28] considered the (three-dimensional) container loading problem and reviewed modeling approaches, as well as exact and heuristic algorithms. A recent survey on multi-dimensional packing problems was presented by Crainic, Perboli, and Tadei [59];

- Integrated variants. Reviews of algorithms for integrated routing and packing problems (with two- and three-dimensional packing constraints) have been presented by Iori and Martello 
[99, 100] and Pollaris et al. [153. Melega, de Araujo, and Jans [135] recently reviewed integrated lot-sizing and cutting stock problems.

The constant interest in cutting and packing problems is shown by:

- the trend of number of publications in the last 20 years, according to the major databases, shown in Figure 1. In particular, the total number of publications in the last 20 years has been 1410, 774, and 575 according to Scholar, Scopus, and WoS, respectively;

- a number of special issues devoted to this area by international journals like, e.g., INFOR (see Martello [125]), European Journal of Operational Research (see Oliveira and Wäscher [147]), International Journal of Production Economics (see Bennell, Oliveira, and Wäscher [21]);

- the recent comprehensive book by Scheithauer [164] dedicated to cutting and packing optimization;

- the visual application for two-dimensional packing problems made available by Costa et al. [55];

- the working group on cutting and packing (ESICUP) of the Association of European Operational Research Societies (EURO), see https://www.euro-online.org/web/ewg/25/.

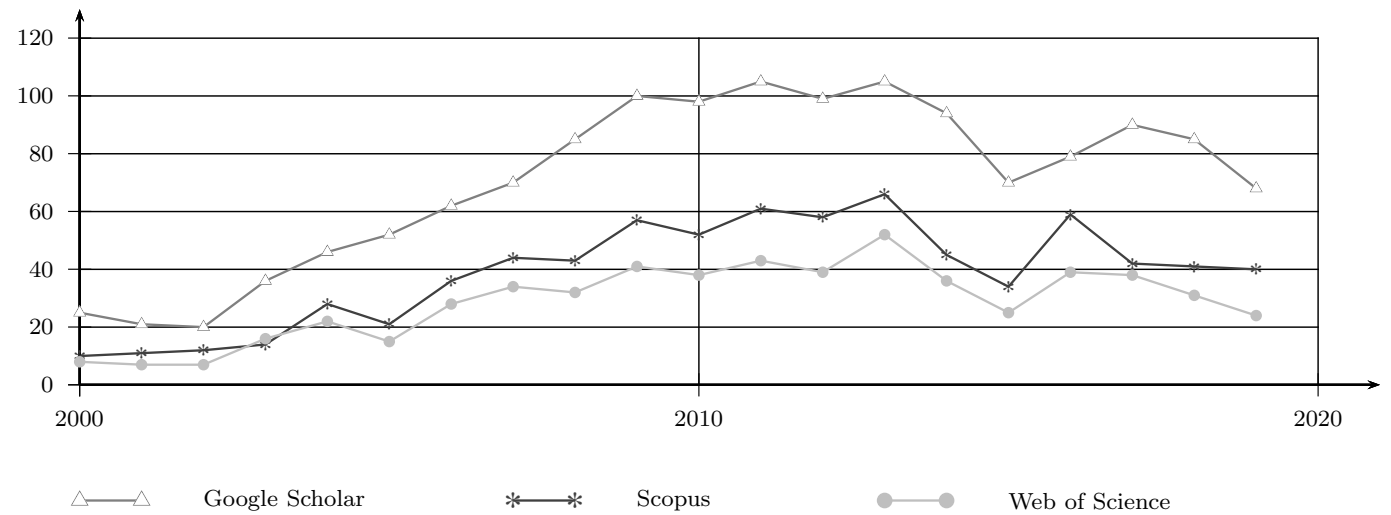

Figure 1: Trend of number of publications on two-dimensional cutting and packing.

\section{Contents}

In this paper, we propose an extensive review of cutting and packing problems of two-dimensional rectangular items. We concentrate in particular on orthogonal cutting and packing, i.e., on the case in which the items must be cut/packed with their edges parallel to those of the bin. Some authors include the term "geometric" in the problem names. The main problems that we address are:

- the two-dimensional strip packing problem: find a packing of minimum height into a single bin with fixed width;

- the two-dimensional bin packing problem: determine the minimum number of bins needed to pack the items;

- the two-dimensional knapsack problem: find a packing of maximum value into a single bin; 
- the two-dimensional orthogonal packing problem: find a feasible item packing (if any) into a single bin.

We also consider some generalizations of these problems, such as the cutting stock problem, as well as relevant variants, e.g., the case in which the items can be rotated by 90 degrees or guillotine cuts are required. We review the main relaxation methods, along with the main heuristics and exact methods.

The literature often considers formulations for two-dimensional cutting and packing problems which are based on a set of points where an item may be packed or cut. We will discuss the main methods for generating such sets of points. Another contribution of our work is the review of preprocessing methods for cutting and packing problems, i.e., methods that are considerably fast, if compared to the overall solution time, and may reduce the size of the instances, thus simplifying their resolution.

The remainder of this survey is organized as follows. Section 2 formally introduces the basic problems we address and the main variants considered in the literature. Section 3 reviews the principal techniques that can be used, in a preprocessing phase, to simplify a problem instance. Section 4 discusses relaxation methods that provide valid bounds and, in some cases, are the starting point to derive a heuristic solution. Section 5 briefly mentions the main heuristic techniques that are frequently embedded into exact approaches (although the scope of this survey is not to extensively review the huge literature on this topic). Section 6 examines mathematical models that explicitly require a solver, by classifying them according to their size (polynomial, pseudo-polynomial, or exponential). Section 7 reviews enumeration schemes that do not explicitly make use of a solver (branch-and-bound, graph-based approaches, and constraint programming). Section 8 provides pointers to relevant unsolved or challenging instances. Finally, Section 9 discusses the state-of-theart for each problem and presents some conclusions and future research directions.

\section{Problems and Definitions}

In this section, we present a formal definition of the main problems and variants we consider. Given a bin and a set of items, a packing consists of a placement of the items such that the items lie completely inside the bin and there is no overlapping between any pair of items.

We are mainly interested in two-dimensional packing problems, in which the bins and the items are rectangles. Only orthogonal packings will be considered, i.e., we require that all edges of all items are parallel to the edges of the bin. Unless otherwise specified, we assume that the items have fixed orientation: as most of the literature considers this case, we will only address as a variant the case in which the items can be rotated by 90 degrees.

A rectangular two-dimensional bin $\mathcal{B}$ is defined by its width $W \in \mathbb{Z}_{+}$and height $H \in \mathbb{Z}_{+}$. We denote by $\mathcal{I}$ a set of rectangular two-dimensional items. Each item $i \in \mathcal{I}$ has width $w_{i} \in \mathbb{Z}_{+}$and height $h_{i} \in \mathbb{Z}_{+}$, such that $0<w_{i} \leq W$ and $0<h_{i} \leq H$. Unless otherwise specified, copies of identical items are treated as distinct items. A packing of $\mathcal{I}$ into $\mathcal{B}$ can be represented by a function $\mathcal{F}: \mathcal{I} \rightarrow \mathbb{Z}_{+}^{2}$ that maps each item $i \in \mathcal{I}$ to a pair $\mathcal{F}(i)=\left(x_{i}, y_{i}\right)$ representing the relative coordinates of the bottom-left corner of the item with respect to the bottom-left corner of the bin. $\mathcal{F}$ is a feasible packing if

$$
\begin{array}{lr}
x_{i} \in\left\{0, \ldots, W-w_{i}\right\} \text { and } y_{i} \in\left\{0, \ldots, H-h_{i}\right\} & (i \in \mathcal{I}) \\
{\left[x_{i}, x_{i}+w_{i}\right) \cap\left[x_{j}, x_{j}+w_{j}\right)=\emptyset \text { or }\left[y_{i}, y_{i}+h_{i}\right) \cap\left[y_{j}, y_{j}+h_{j}\right)=\emptyset} & (i, j \in \mathcal{I}, i \neq j) .
\end{array}
$$

In other words, the bin is seen on a Cartesian plane with its edges parallel to the $x$ and $y$ axes and its bottom-left corner on the origin. A packing defines, for every item $i$, the coordinates $\left(x_{i}, y_{i}\right)$ 
where its bottom-left corner is placed. Constraints (1) impose that each item is entirely inside the bin, while constraints (2) forbid overlapping between any pair of items.

\subsection{Problems}

In this section, we define the main two-dimensional orthogonal packing problems we consider. As mentioned in Section 1, some typologies have been proposed in the literature to classify the great variety of possible packing problems. As we will mainly deal with the four problems defined below, we preferred to use the simple names we provide. For the sake of completeness, their definition is followed, in Section 2.2, by the corresponding notation in the main typologies. A simple visual example of a set $\mathcal{I}$ containing 10 items is given in Figure 2(a), and then used to clarify the main problem variants below.

\section{Two-Dimensional Strip Packing Problem}

We are given a single bin $\mathcal{B}$ having fixed width $W$ and infinite height, usually called a strip. The Two-Dimensional Strip Packing Problem (2D-SPP) asks for a feasible packing of $\mathcal{I}$ into $\mathcal{B}$ such that the height at which the strip is used (i.e., the height of the topmost edge of an item) is minimized. Figure 2(b) shows a minimum height arrangement of the items of Figure 2(a) into a strip.

\section{Two-Dimensional Bin Packing Problem}

In this case we have an unlimited number of identical finite bins $\mathcal{B}$ having width $W$ and height $H$. The Two-Dimensional Bin Packing Problem (2D-BPP) requires to determine a partition of $\mathcal{I}$ into the minimum number of subsets, such that each subset can be feasibly packed into a bin. A generalization of the problem is the Two-Dimensional Cutting Stock Problem (2D-CSP), in which one is asked to pack a given number $d_{i}$ (demand) of each item $i \in \mathcal{I}$. Both problems generalize their one-dimensional version, the well-known (one-dimensional) Bin Packing Problem (1D-BPP) and Cutting Stock Problem (1D-CSP), in which the items are segments of size $w_{i}(i \in \mathcal{I})$ and the bins are segments of size $W$ (capacity). An extensive literature exists on these problems, see 65] for a recent survey. Figure 2(c) shows an optimal 2D-BPP solution, in which the items of Figure 2 (a) are packed into two separate bins.

\section{Two-Dimensional Knapsack Problem}

The problems listed so far ask for a feasible packing of all items of $\mathcal{I}$. Assume now that every item $i \in \mathcal{I}$ has an associated value (profit) $v_{i} \in \mathbb{Z}_{+}$. The Two-Dimensional Knapsack Problem (2D-KP) requires to determine a subset of items $\mathcal{I}^{\prime} \subseteq \mathcal{I}$ such that: (i) there exists a feasible packing of $\mathcal{I}^{\prime}$ into a single bin $\mathcal{B}$; and (ii) the corresponding total profit, $\sum_{i \in \mathcal{I}^{\prime}} v_{i}$, is maximized. A special case of the 2D-KP, the Two-Dimensional Rectangular Packing Problem, arises when the profit of each item is equal to its area (i.e., $v_{i}=w_{i} h_{i} \forall i \in \mathcal{I}$ ). An example of an optimal two-dimensional rectangular packing problem solution for the items of Figure 2(a) is given in Figure 2(d).

\section{Two-Dimensional Orthogonal Packing Problem}

While the above problems are in optimization version, the Two-Dimensional Orthogonal Packing Problem (2D-OPP) is to decide if there exists a feasible packing of a given set $\mathcal{I}$ of items into a single bin $\mathcal{B}$. Although this problem is in decision version, i.e., it just asks for a 'yes/no' answer, in practical applications one is generally requested to also produce the specific packing, if any. The 2D-OPP appears as a subproblem in the optimization version of a number of packing problems. 


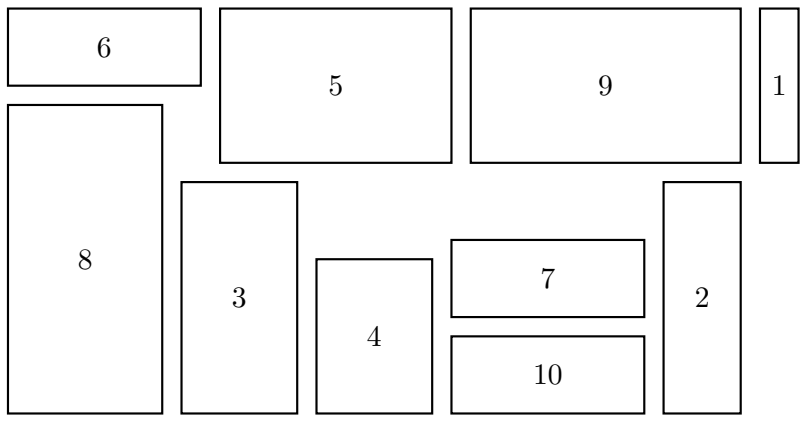

(a)
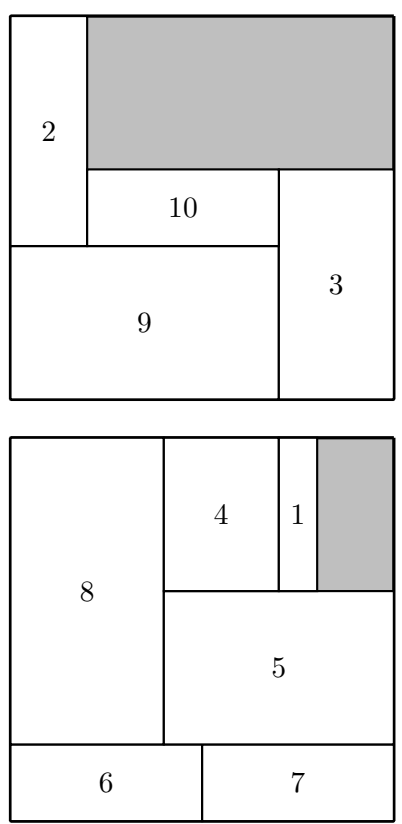

(c)

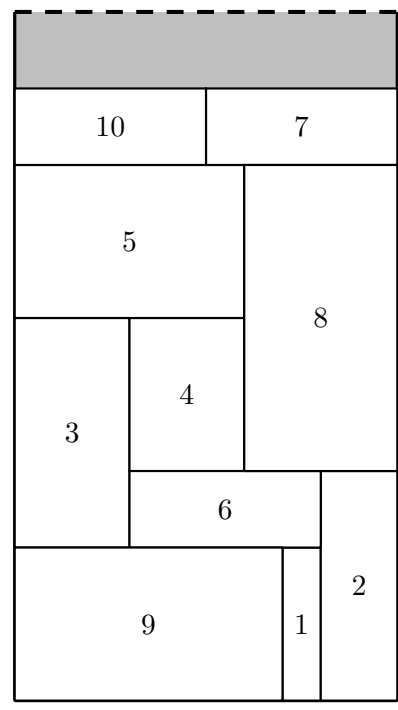

(b)

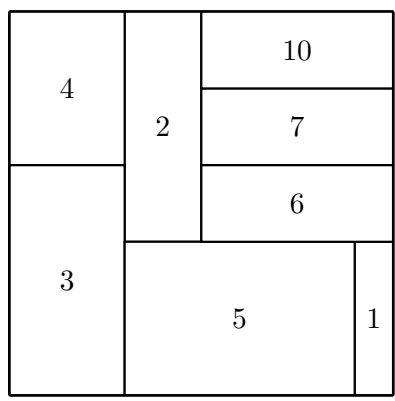

(d)

Figure 2: (a) set of items; (b) an optimal 2D-SPP solution; (c) an optimal 2D-BPP solution; (d) an optimal 2D-KP solution (in case items profits correspond to their areas).

\subsection{Typologies}

As previously mentioned, a number of typologies have been introduced in the literature. As the reader could encounter the same problems considered in the present survey but identified in a different way, we next provide the notations adopted by the most common typologies from the literature.

According to the typology proposed by Dyckhoff [71] in 1990, the 2D-BPP, the 2D-CSP and the $2 \mathrm{D}-\mathrm{KP}$ are denoted as $2 / \mathrm{V} / \mathrm{I} / \mathrm{M}, 2 / \mathrm{V} / \mathrm{I} / \mathrm{R}$, and $2 / \mathrm{B} / \mathrm{O} /$, respectively. The $2 \mathrm{D}-\mathrm{SPP}$ and the $2 \mathrm{D}-\mathrm{OPP}$ have not been formally defined in this typology.

In 1999 Lodi, Martello, and Vigo [119] used a three-field typology (later extended by Martello, Monaci, and Vigo [128]). The 2D-SPP, the 2D-BPP, and the 2D-KP are denoted as $2 \mathrm{SP}|\mathrm{O}| \mathrm{F}$, 
$2 \mathrm{BP}|\mathrm{O}| \mathrm{F}$, and $2 \mathrm{KP}|\mathrm{O}| \mathrm{F}$, respectively, while the $2 \mathrm{D}-\mathrm{OPP}$ is not classified. The second field of this notation also covers the variant (see below) in which orthogonal rotation of the items is allowed (' $\mathrm{R}$ ' instead of ' $\mathrm{O}$ '), while the third field can handle the variant in which guillotine cuts are required ('G' instead of ' $F$ ').

More recently (2007), Wäscher, Haußner, and Schumann [178] proposed a successful typology, partially based on Dyckhoff's original ideas. According to it, the 2D-SPP is denoted as the "(Twodimensional) Open Dimension Problem" (ODP), the 2D-BPP as the "Single Bin Size Bin Packing Problem" (SBSBPP), and the 2D-CSP as the "Single Stock Size Cutting Stock Problem" (SSSCSP). The 2D-KP is denoted either as the "Single Knapsack Problem" (SKP), or as the "Single Large Object Placement Problem" (SLOPP) in the generalization in which each item $i \in \mathcal{I}$ is available in $d_{i}$ copies. The 2D-OPP is not explicitly classified in [178], but it can be interpreted as the recognition version of the $2 \mathrm{D}-\mathrm{BPP}$.

\subsection{Complexity}

The one-dimensional version of the $2 \mathrm{D}-\mathrm{KP}$ is the well-known knapsack problem (1D-KP) in which each item has a weight $w_{i}$ and the bin (knapsack) has capacity $W$. The $1 \mathrm{D}-\mathrm{KP}$ can be solved in pseudo-polynomial time through dynamic programming (see, e.g., Martello and Toth [130] or Kellerer, Pferschy, and Pisinger [109]). Instead, none of the above two-dimensional problems admits a pseudo-polynomial time algorithm, unless $\mathcal{P}=\mathcal{N} \mathcal{P}$. Recall indeed that the $1 \mathrm{D}$-BPP is strongly $\mathcal{N} \mathcal{P}$-hard (see Garey and Johnson [84]). Given an instance of the 1D-BPP, define two-dimensional items having width $w_{i}$ and height $h_{i}=1(i \in \mathcal{I})$. Then:

- the solution of a 2D-SPP instance with strip width $W$ solves the 1D-BPP instance;

- the solution of a 2D-BPP instance with bins of width $W$ and height 1 solves the 1D-BPP instance;

- associate a profit $v_{i}=1$ to each item $i \in \mathcal{I}$. The minimum value $\bar{H}$ for which the optimal solution of a 2D-KP instance with a bin of width $W$ and height $\bar{H}$ has value $n$ gives the optimal 1D-BPP solution value;

- the solution of a 2D-OPP instance with a bin of width $W$ and height $k$ answers the decision version of the 1D-BPP: can the items of $\mathcal{I}$ be packed into $k$ bins?

It follows that the existence of a polynomial-time algorithm for any of the four problems above would imply a polynomial-time algorithm for the $1 \mathrm{D}-\mathrm{BPP}$, i.e., the 2D-SPP, the 2D-BPP, and the $2 \mathrm{D}$-KP are strongly $\mathcal{N} \mathcal{P}$-hard, and the $2 \mathrm{D}$-OPP is strongly $\mathcal{N} \mathcal{P}$-complete.

\subsection{Variants}

Motivated by practical applications, a number of variants of two-dimensional packing problems has been considered in the literature (see, e.g., Lodi, Martello, and Vigo [119], Pisinger and Sigurd [151, and Wäscher, Haußner, and Schumann [178]).

\section{Orthogonal Rotation}

While in the basic problems the items have fixed orientation, relevant variants allow item rotation by 90 degrees. For example, while in the cutting of corrugated or decorated stock units rotation is forbidden, when the surfaces are uniform it may be feasible to rotate the items in order to produce better (more dense) packings. Note however that this also leads to more complex problems as it 
increases the number of decisions to be taken, and hence the corresponding models usually involve a higher number of variables and constraints. Indeed, in most approaches, item rotation is typically handled either by adding, for each item, a "rotate-or-not" binary decision (like, e.g., in Jakobs [104]), or by creating a companion (rotated) copy of each item and forbidding that both are selected (like, e.g., in Lodi and Monaci [123]).

\section{Guillotine Cuts}

Normally, automatic cutting machines can only produce the items through a sequence of guillotine cuts, i.e., edge-to-edge cuts parallel to the edges of the bin. Imposing such constraint may lead to worse solutions as not all item sets allow guillotine patterns in a bin (see Figure 3(a)). Frequently, the machines are restricted to only alternate horizontal and vertical cuts, possibly with a hard limit $k$ on the number of cuts per bin ( $k$-staged problems). In most applications $k$ is two or three, possibly allowing an extra cut (called trimming) to separate an item from waste (see Figure 3 (b) and (c)). When $k=2$, the problem is frequently called level packing, as it can be seen as the problem of packing the items side-by-side on horizontal shelves having width equal to that of the bin/strip and height coinciding with the tallest packed item (see again Figure $3(\mathrm{~b})$ ). When $k=3$, Puchinger and Raidl [154] distinguish between the case where the latter condition holds (restricted case) and the one where the height of a shelf is not necessarily given by its highest item (unrestricted case). The presence of guillotine constraints consistently affects the combinatorial structure of the problem, and hence the solution techniques. Indeed, for such cases, it is common to adopt techniques based on column generation and/or dynamic programming, that would not be suitable for standard non-guillotine problems.

\section{Variable-Sized Bins}

The bin packing and cutting stock problems consider an unlimited number of identical bins. The variable-sized generalization of these problems deals instead with different types of bins, each having a specific size (width and height), cost, and availability. The problem is then to pack all the items at minimum cost. The addition of variable sizes is usually handled in the solution techniques by considering, e.g., the different sizes of the bins when creating new nodes in enumerative approaches, or when pricing variables in branch-and-price methods (as, e.g., in Pisinger and Sigurd [151]). This comes at the cost of an additional computational effort, and indeed, from a practical point of view, the solution of variable sized problems is typically more challenging.

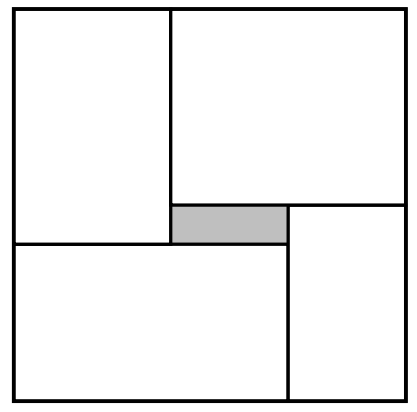

(a)

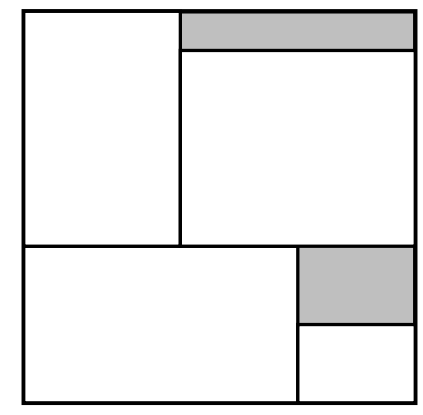

(b)

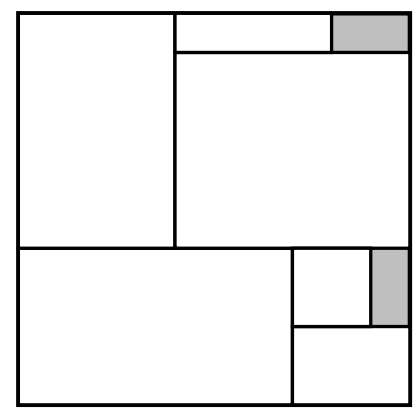

(c)

Figure 3: (a) non-guillotine pattern; (b) 2-staged guillotine pattern with trimming; (c) 3-staged guillotine pattern with trimming. 


\section{Loading and Unloading Constraints}

Loading and/or unloading constraints often arise in applications where goods pertaining to different customers have to be loaded into the same bin, which represents, e.g., the loading area of a truck (see, e.g., Gendreau et al. [85]). In these cases, the vehicles have a loading/unloading orientation, and the sequence of visit to the customers must be such that each item may be moved in/out without moving any other item. These constraints can be directly included in mathematical models where the variables indicate the position of the items (see Section 6.2) as the packing of an item restricts the range of positions that can be taken by other items (as, e.g., in Côté, Gendreau and Potvin [57]). They can also be imposed within branch-and-bound algorithms, where additional fathoming criteria can be devised to reduce the number of decision nodes (as, e.g., in Iori, Salazar González and Vigo [102]).

The first two variants were already discussed in the Sixties by Gilmore and Gomory [88. In the Eighties, Friesen and Langston [79] introduced (for the one-dimensional case) the variable-size variant. The studies on loading and unloading constraints started in the Noughties, see Gendreau et al. [85.

\section{Sets of Points and Preprocessing Techniques}

We start this section by reviewing techniques based on sets of points, that can be used in both Integer Linear Programming (ILP) models and solution methods reviewed in the following sections. We then examine some preprocessing techniques, used to decrease the size of a given instance.

\subsection{Sets of Points}

Several authors in the literature use sets of points to represent the possible positions where an item can be packed. According to the definitions given in Section 2, packing an item at a point $p=(x, y)$ means to allocate the item into the bin with its bottom-left corner in the position identified by $p$.

As we assume that items and bins have integer sizes, only considering integer point coordinates does not affect optimality. Trivial sets of points for an item $i$ are thus given by coordinate sets $X_{i}=\left\{0, \ldots, W-w_{i}\right\}$ for the $x$-axis and $Y_{i}=\left\{0, \ldots, H-h_{i}\right\}$ for the $y$-axis.

It is clear that the smaller the number of points the smaller the search domain. We review in the following the main techniques that have been proposed in the literature for generating reduced sets of points by preserving optimality.

Already in the Seventies, Herz [95] and Christofides and Whitlock [45] independently observed that any feasible packing pattern can be transformed into an equivalent one (normal pattern) in which the items are shifted to the bottom and left as much as possible. In a normal pattern, the left and bottom edges of each item touch another item or the bin. This leads to the definition of the set of "normal" $x$-coordinates

$$
\mathcal{N}^{\mathrm{x}}=\left\{x \mid x<W, x=\sum_{i \in \mathcal{I}} w_{i} \zeta_{i}, \zeta_{i} \in\{0,1\}(i \in \mathcal{I})\right\}
$$

defining the only $x$-coordinates at which an item can be packed. A similar definition holds for the set $\mathcal{N}^{\mathrm{y}}$ of normal $y$-coordinates.

Boschetti, Hadjiconstantinou, and Mingozzi [29] proposed to separately compute a set of normal patterns for each item $k \in \mathcal{I}$ by excluding those patterns that include $k$ itself. The resulting set $\mathcal{N}_{k}^{\mathrm{x}}(k \in \mathcal{I})$ is obtained from $(3)$ by replacing $\mathcal{I}$ with $\mathcal{I} \backslash\{k\}$. (Similarly for $\mathcal{N}_{k}^{\mathrm{y}}$.) 
Terno, Lindemann, and Scheithauer [171] proposed the use of reduced raster points, obtained by removing redundant positions from the set of normal patterns. The idea is that, given two coordinates $p, q \in \mathcal{N}^{\mathrm{x}}(p<q)$, if every possible combination of items that can be packed to the right of $p$ can also be packed to the right of $q$, then $p$ can be removed from $\mathcal{N}^{\mathrm{x}}$.

Recently, Côté and Iori [58] proposed a new set of patterns called meet-in-the-middle, generated by first defining a threshold $t \in\{1,2, \ldots, W\}$ and then left-aligning patterns that are to the left of $t$ and right-aligning those that are to its right. The resulting set of patterns is proved to be never larger than $\mathcal{N}^{\mathrm{x}}$, and in practice is usually much smaller.

The fact that normal patterns preserve optimality was proved in the seminal paper by Herz 95]. Proofs were later provided for other types of patterns like, e.g., in Côté and Iori [58]. All such proofs show that any solution in which the items are packed without restrictions in the continuous two-dimensional space can be transformed, through simple translations, into a better or equivalent solution in which the items are packed according to the patterns. The same arguments apply to problem variants like, e.g., those involving guillotine cuts or several item copies or item rotation.

\subsection{Preprocessing Techniques}

Two main approaches for preprocessing the instances by preserving optimality can be found in the literature: methods that fix some decisions and methods that modify the input parameters. These techniques are useful for improving bounds based on the area of the items or of the bins. We describe in the following one method of the former type and two of the latter. Although developed

for specific problems, the methods of the latter type can be directly used for all problems. The extension of methods of the former type is instead not straightforward.

All methods will be presented referring to widths. Those developed for problems on bins can be identically applied using heights instead of widths, while the same does not hold for problems on strips. The sequence of presentation follows the order in which it is advisable to execute these methods in order to effectively reduce the given instance.

\section{Packing a Subset of Items}

This reduction was originally proposed by Martello, Monaci, and Vigo [128] for the 2D-SPP. Suppose that the items are sorted by non-increasing width. The method can be applied if $w_{1}>W / 2$. Let $B=\left\{i \in \mathcal{I} \mid w_{i}=w_{1}\right\}$. Observe that these items cannot be packed side-by-side, so we can pile them aligned to the left edge of the strip. Now define the set of those items that can be packed side-by-side with an item of $B$, namely $S=\left\{j \mid w_{j} \leq W-w_{1}\right\}$. If there exists a feasible packing of all the items of $S$ into the empty right part of the strip, with overall height not greater than $\sum_{i \in B} h_{i}$, then this packing can be optimally fixed on the bottom of the strip, and the process can be iterated on the reduced instance. When the required packing is not found, the method finds the first item $\ell \notin B$. If $w_{\ell}>W / 2$, the current set $B$ is updated by adding the items of width $w_{\ell}$, the current set $S$ is updated accordingly, and a new attempt is performed.

This reduction was later used within other solution methods for the 2D-SPP (see, e.g., AlvarezValdes, Parreño, and Tamarit [5], Boschetti and Montaletti [32] and Côté, Dell'Amico, and Iori [56]) and extended to other packing problems, among which the 2D-BPP (see, e.g., Carlier, Clautiaux, and Moukrim [49]).

\section{Shrinking the Size of the Bin}

Alvarez-Valdes, Parreño, and Tamarit [5] proposed to solve a (one-dimensional) subset-sum problem (find a subset of a set of given integers whose sum is closest to, without exceeding, a prefixed 
threshold) to determine the maximum value $\bar{W} \leq W$ such that there exists a sum of item widths equal to $\bar{W}$. If $\bar{W}<W$ then the width of the bin/strip can be reduced to $\bar{W}$.

\section{Lifting the Size of the Items}

A similar approach had been used by Boschetti, Hadjiconstantinou, and Mingozzi [29] to increase item widths. They proposed to solve, for each item $i \in \mathcal{I}$, a subset-sum problem to determine the maximum value $\bar{W}_{i} \leq W-w_{i}$ such that there exists a set of items in $\mathcal{I} \backslash\{i\}$ with total width equal to $\bar{W}_{i}$. If $w_{i}+\bar{W}_{i}<W$ then the width of $i$ can be increased by $W-\bar{W}_{i}$. Carlier, Clautiaux, and Moukrim [49] defined similar methods for updating the size of the items by removing small items and increasing the width of large items.

\section{Relaxations}

Several relaxation methods for two-dimensional packing problems have been proposed in the literature. They are used within exact algorithms and, in some cases, as a base to construct a heuristic solution. Obviously, relaxations provide lower bounds for the 2D-SPP and the 2D-BPP and upper bounds for the 2D-KP. As the 2D-OPP is a decision problem, relaxations can be used to prove that the required packing does not exist.

\subsection{Continuous Relaxation}

Splitting each item $i$ into $w_{i} \times h_{i}$ unit squares produces the most immediate relaxation for all considered problems. For the 2D-OPP, the 2D-SPP and the 2D-BPP, the continuous lower bound is then

$$
\left\lceil\sum_{i \in \mathcal{I}} \frac{w_{i} h_{i}}{W H}\right\rceil
$$

(computed with $H=1$ for the 2D-SPP). For the 2D-KP, the continuous upper bound is obtained, following Dantzig [63] by: (i) sorting the items by non-increasing ratios $v_{i} /\left(w_{i} h_{i}\right)$; (ii) finding the first item $s$ such that $\sum_{i=1}^{s} w_{i} h_{i}>W H$ and defining $c=W H-\sum_{i=1}^{s-1} w_{i} h_{i}$; (iii) computing the upper bound as

$$
\left\lfloor\sum_{i=1}^{s-1} v_{i}+\frac{c}{w_{s} h_{s}} v_{s}\right\rfloor .
$$

Efficient implementations allow all these bounds to be computed in linear time. Because of its simplicity, the continuous relaxation has been used in almost all works on two-dimensional packing problems.

\subsection{Combinatorial Bounds}

Martello and Vigo 131 extended to the 2D-BPP some lower bounds proposed by Martello and Toth [129] for the 1D-BPP and by Dell'Amico and Martello [64] for the $P \| C_{\max }$, a parallel machine scheduling problem that is strictly related to the 1D-BPP. The idea is to identify, for a given parameter $p$ : (i) two item sets, say $J_{1}(p)$ and $J_{2}(p)$, such that no two items of $J_{1}(p) \cup J_{2}(p)$ may be packed into the same bin; (ii) a third set, $J_{3}(p)$, of items that cannot be packed into a bin used for an item of $J_{1}(p)$. A valid lower bound is then $L(p)=\left|J_{1}(p) \cup J_{2}(p)\right|+L_{23}(p)$, where $L_{23}(p)$ denotes a lower bound on the number of additional bins needed for the items of $J_{3}(p)$. It is shown that the overall lower bound, $\max _{p}\{L(p)\}$ can be computed in $O\left(n^{2}\right)$ time. 
Boschetti and Mingozzi [30, 31] improved these bounds and extended them to the variant in which orthogonal rotation of the items is allowed. Similarly, relaxations for the 2D-KP can be obtained from induced 1D-KP instances where each item has a weight equal to its area and the knapsack has capacity $W H$.

Although combinatorial bounds have a low computational cost, they can be quite effective, especially in branch-and-bound approaches (see, e.g., Martello, Monaci, and Vigo [128] and AlvarezValdes, Parreño and Tamarit [5]).

\subsection{Linear Relaxation and Column Generation}

Already in 1965, the seminal paper by Gilmore and Gomory [88 introduced a column generation algorithm for two-dimensional packing problems. The algorithm is based on the linear relaxation of a mathematical formulation (the so-called set covering model) that they had developed for the 1D-CSP (see Section 2.1). The model has a variable for each possible combination of items that fits into a single bin (pattern). Since the resulting Linear Program (LP) has a huge (exponential) number of columns (variables), the Column Generation method starts with a restricted model that only includes a subset of columns and iteratively adds columns that may improve the current solution. This relaxation has been used in branch-and-price algorithms for the 2D-BPP and related variants (see, e.g., Pisinger and Sigurd [151, 152]), and in problems involving guillotine cuts (see, e.g., Belov and Scheithauer [19, Bettinelli, Ceselli, and Righini [25], and Cintra et al. [46]).

\subsection{Dual Feasible Functions}

Dual feasible functions were originally introduced by Johnson [106] for a normalized version of the $1 \mathrm{D}-\mathrm{BPP}$ in which the bin capacity is 1 and the item sizes $w_{i}$ are values in $[0,1]$.

A function $f:[0,1] \rightarrow[0,1]$ is a Dual Feasible Function (DFF), if for any finite set $S$ of non-negative real numbers, it holds that:

$$
\sum_{x \in S} x \leq 1 \Longrightarrow \sum_{x \in S} f(x) \leq 1
$$

Three classes of DFFs were proposed by Fekete and Schepers [72]. The survey by Clautiaux, Alves, and Valério de Carvalho [47] describes relevant (superadditive and maximal) DFFs that can be used in packing contexts. Rietz, Alves, and Valério de Carvalho [158, 159, 160], analyzed a number of properties of DFFs (maximality, extremality, worst-case performance) and studied the best parameter tuning for a specific function.

Dual feasible functions can be extended to consider discrete domains and used to derive relaxations for higher-dimensional cutting and packing problems (see Fekete and Schepers [74]). For the 2-dimensional case, let $f$ and $g$ be two DFFs. Applying $f$ to the item widths and $g$ to the item heights of an instance of any two-dimensional packing problem, we obtain a new instance whose relaxations are valid for the original instance.

Carlier, Clautiaux, and Moukrim [49] proposed DFF-based lower bounds for the 2D-BPP and introduced DFFs depending on the sizes of items and bins (data-dependent DFFs). Caprara and Monaci [37] solved a bilinear programming problem to determine DFFs that provide the best bound for a given two-dimensional instance.

Fekete and Schepers [74] extended the concept of DFF to that of conservative scale. A conservative scale is a set of modified item sizes $\widetilde{w}$ (resp. $\widetilde{h}$ ) such that any subset $\mathcal{I}^{\prime}$ of items satisfying

$\sum_{i \in \mathcal{I}^{\prime}} w_{i} \leq W\left(\operatorname{resp} . \quad \sum_{i \in \mathcal{I}^{\prime}} h_{i} \leq H\right)$ also satisfies $\sum_{i \in \mathcal{I}^{\prime}} \widetilde{w}_{i} \leq W\left(\operatorname{resp} . \quad \sum_{i \in \mathcal{I}^{\prime}} \widetilde{h}_{i} \leq H\right)$. Note that a conservative scale is instance dependent while dual feasible functions have general validity. 


\begin{tabular}{|c|c|c|c|}
\hline 6 & 7 & 5 & \\
\hline 6 & 3 & 5 & \\
\hline 6 & 3 & 5 & \\
\hline 6 & 3 & 5 & \\
\hline 1 & 3 & & \\
\hline 1 & 3 & & \\
\hline 1 & 3 & & \\
\hline 1 & 3 & & \\
\hline 1 & 2 & 4 & \\
\hline 1 & 2 & 4 & \\
\hline
\end{tabular}

(a)

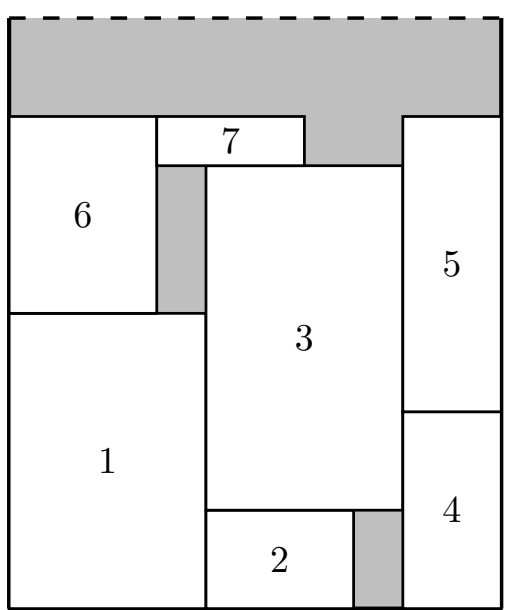

(b)

Figure 4: (a) contiguous relaxation of items 1-7; (b) two-dimensional packing obtained from (a).

The relationship between (data-dependent) dual feasible functions and conservative scales has been deeply investigated by Belov et al. [17].

Recently, Serairi and Haouari [165] presented a theoretical and experimental study of the most important polynomial-time lower bounding procedures for the 2D-BPP. Among the algorithms they tested, the one by Carlier, Clautiaux, and Moukrim [49] turned out to provide the tightest values.

For a comprehensive study on the use of dual feasible functions for integer programming and combinatorial optimization problems the reader is referred to the recent book by Alves et al. [6]. Due to their computational efficiency, dual-feasible functions are often implemented in branch-andbound algorithms (see, e.g., Clautiaux, Carlier, and Moukrin [49], and Alvarez-Valdes, Parreño and Tamarit [5]).

\subsection{Contiguous One-Dimensional Relaxations}

In order to obtain a relaxation of the 2D-SPP, Martello, Monaci, and Vigo [128 introduced the One-dimensional Contiguous Bin Packing Problem (1D-CBPP). The idea is to horizontally "cut" each item $i \in \mathcal{I}$ into $h_{i}$ unit-height slices of width $w_{i}$ and to solve a 1D-BPP problem with capacity $W$ : the resulting number of one-dimensional bins is then a lower bound on the height of any 2D-SPP solution. To tighten the bound, the 1D-CBPP additionally imposes contiguity: all slices obtained from an item $i$ must be packed into $h_{i}$ consecutive one-dimensional bins (see Figure 4 (a)). Although the $1 \mathrm{D}-\mathrm{CBPP}$ is a relaxation of the $2 \mathrm{D}$-SPP, it remains strongly $\mathcal{N} \mathcal{P}$-hard and hence it can only be optimally solved through enumeration. A branch-and-bound algorithm was presented in [128] for its exact solution and the resulting lower bound was used in an enumerative algorithm for the 2D-SPP. Later, Alvarez-Valdes, Parreño, and Tamarit [5] proposed an ILP model for the 1D-CBPP, and used a commercial software to solve it within a branch-and-bound algorithm for the 2D-SPP.

Belov et al. [16] used the 1D-CBPP as a relaxation of the 2D-OPP. They proposed an alternative ILP model for the 1D-CBPP and a weaker relaxation in which, instead of requiring contiguity, it is imposed that each one-dimensional bin can contain at most one slice from each item (bar relaxation). Friedow and Scheithauer [78] solved the 1D-CBPP with a cutting plane approach based on column generation for the bar relaxation. A branch-and-bound algorithm for the 1D-CBPP was later proposed by Mesyagutov et al. [138]. 
Côté, Dell'Amico, and Iori [56] proposed an algorithm for the 2D-SPP based on Benders' decomposition (see Section 6.3 below) of an ILP model. The resulting master problem, obtained by "transposing" the instance and vertically cutting the items into unit-width bars, is a parallel processor scheduling problem with contiguity constraints. The slave problem, which is still strongly $\mathcal{N} \mathcal{P}$-hard, determines whether the solution provided by the master can be transformed into a feasible packing (see Figure 4(b)). Delorme, Iori, and Martello [66] applied a similar decomposition to solve the 2D-SPP with orthogonal rotations. They proposed an ILP model for the 1D-CBPP based on the classical arc-flow formulation of the 1D-CSP (see Valério de Carvalho [172]).

\subsection{State Space Relaxations}

The state space relaxation, originally developed by Christofides, Mingozzi, and Toth [44 for routing problems, modifies the state space of a dynamic programming recursion so that its optimal solution is a valid bound for the original problem. This relaxation has been used for the (strongly NP-hard) guillotine $2 \mathrm{D}-\mathrm{KP}$, for which a state space relaxation can disregard the constraint on the maximum number of copies of each item. The relaxed problem (known in the literature as the unconstrained two-dimensional guillotine knapsack problem) can be solved in pseudo-polynomial time through dynamic programming (see, e.g., Gilmore and Gomory [88, Beasley [12], Cintra et al. [46] and Russo et al. [162]).

Christofides and Hadjiconstantinou [43] strengthened the state space relaxation by associating a non-negative integer multiplier with each item and adopted a subgradient-like procedure to determine good multipliers. Improvements were proposed by Morabito and Pureza [141] and by Velasco and Uchoa [176].

\section{Heuristics}

There is a huge literature on approximation algorithms and heuristics for two-dimensional packing problems, and a thorough review of these methods is outside the scope of this survey. To get an idea of the vastity of this area, it is enough to consider that Ortmann and van Vuuren [149] computationally compared a total of 252 heuristics and variants for two-dimensional strip packing problems. As heuristics are frequently used within exact algorithms (e.g., to initialize the incumbent solution), we provide in the following some pointers to a number of relevant results.

\subsection{Approximation and On-line Algorithms}

Concerning approximation and on-line algorithms, we refer the reader to the recent (2017) comprehensive survey by Christensen et al. [42], that gives theoretical insight on fast solution methods characterized by relevant theoretical properties for multi-dimensional packing problems. We only mention here recent relevant works that appeared after the publication of such survey.

Gálvez et al. 83 proposed a polynomial-time 1.89-approximation algorithm for the 2D-KP with orthogonal rotations, breaking the previous 2-approximation barrier.

$\mathrm{Yu}$, Mao, and Xiao [184, 186] studied the on-line 2D-SPP, presenting new lower and upper bounds with guaranteed worst-case performance. In [185] they presented an upper bound for the special case of the 2D-SPP with square items. Further improvements were presented by Han et al. 93. for the 2D-SPP and by Balogh et al. [11] for the special case of the 2D-BPP in which items are squares.

For the 2D-SPP, Henning et al. 94] studied pseudo-polynomial approximation algorithms with respect to the width of the strip, i.e., algorithms whose time complexity is a polynomial function of 
the strip width. They proved that there cannot exist a pseudo-polynomial algorithm with a ratio better than $5 / 4$ unless $\mathcal{P}=\mathcal{N} \mathcal{P}$. Jansen and Rau [105] closed the gap between inapproximability result and best known algorithm by presenting an algorithm with approximation ratio $5 / 4+\varepsilon$.

\subsection{Constructive Heuristics, Local Search, and Metaheuristics}

A huge number of constructive heuristics for two-dimensional cutting and packing problems can be found in the literature. The reader is referred to Lodi et al. [118 for classical results in this area. We only mention here two strategies that have been the basis of many solution approaches. The classical bottom-left algorithm, proposed in 1980 by Baker, Coffman, and Rivest [9], packs one item at a time in the lowest possible position, left justified. Another classical result (the bestfit approach), proposed by Burke, Kendall, and Whitwell [35], selects and packs an item that better fits the lowest available area. (See Chazelle [40] and Imahori and Yagiura 98] for efficient implementations of these methods.)

Local search approaches explore the neighborhood of a given solution. Several methods for twodimensional packing problems are based on fixing part of the solution, unpacking the other items, and completing the solution according to some strategy. For example, Alvarez-Valdes, Parreño, and Tamarit [3] create "holes" in the current packing and try to use them when completing the solution. Other approaches pack items according to an input sequence, and perform changes in such sequence to obtain alternative solutions (see, e.g., Burke, Hyde, and Kendall [34] and Wei et al. [180]).

Constructive heuristics and local search are the base of many efficient metaheuristic algorithms. We briefly review in the following some results for the two main metaheuristic approaches.

\section{Single-solution Metaheuristics}

Alvarez-Valdés, Parajón, and Tamarit [1] proposed a GRASP and a Tabu search for the 2D-KP with guillotine constraints. Alvarez-Valdes, Parreño, and Tamarit [3, 4] presented a Tabu search algorithm for the 2D-KP and a GRASP for the 2D-SPP. Wei et al. [179] proposed an effective Tabu search for the two-dimensional rectangular packing problem (see Section 2.1), both with and without orthogonal rotation. They also embedded it in a binary search approach to solve the corresponding versions of the 2D-SPP.

Some of the metaheuristics proposed in the literature also work for the three-dimensional generalization of the 2D-BPP, in which items and bins are three-dimensional boxes. For both dimensions, Parreño et al. [150] presented a combination of GRASP and variable neighborhood search, while Lodi, Martello, and Vigo [122] implemented a unified C computer code based on Tabu search (available at http://or.dei.unibo.it/research_pages/ORcodes/TSpack.html).

Tabu search heuristics for routing problems with two- and three-dimensional loading/unloading constraints have been proposed by Gendreau et al. [85, 86]

\section{Population-Based Metaheuristics}

Concerning the 2D-SPP, Iori, Martello, and Monaci [101 developed a hybrid Tabu search-genetic algorithm. Different genetic approaches were presented by Burke et al. [33], Matayoshi [134], and Borgulya [27].

Genetic algorithms for the 2D-KP were proposed by Hadjiconstantinou and Iori [92] and by Kierkosz and Luczak [111]. Gonçalves and Resende [89] presented a biased random key genetic algorithm for the $2 \mathrm{D}-\mathrm{BPP}$ and its three-dimensional generalization. 


\subsection{Set covering based heuristics}

Monaci and Toth [139] developed algorithms for the 2D-BPP based on the set-covering formulation (see Section 4.3) induced by a heuristic generation of the patterns.

Cintra et al. [46] proposed dynamic programming algorithms for the guillotine 2D-KP, both in the $k$-staged and in the non-staged versions. They then used these algorithms in a column generation heuristic for the corresponding versions of the 2D-CSP and the 2D-SPP. Other heuristics based on column generation for the 2-staged 2D-CSP were presented by Furini et al. [81, by Cui and Zhao [60] and by Cui, Zhou and Cui [61].

For the 3-staged 2D-CSP, a set covering based heuristic was presented by Vanderbeck [175].

\section{Exact Methods based on Integer Linear Programming Models}

In this section we review solution approaches that are based on ILP and Mixed Integer Linear Programming (MILP) formulations and hence require the use of a solver. The models may be classified on the basis of their size: polynomial, pseudo-polynomial, or exponential.

\subsection{Polynomial Models}

A mathematical mixed-integer model for a general three-dimensional bin packing problem, involving a polynomial number of variables and constraints, was presented by Chen, Lee, and Shen [41. (This obviously implies a polynomial model for the two-dimensional classical problems.) The model in [41, that is based on the enumeration of all possible relative placements of each pair of items, can be seen as an extension to the three-dimensional case of the modeling technique proposed by Onodera, Taniguchi, and Tamaru [148] for a two-dimensional block placement problem.

Polynomial formulations for 2-staged guillotine packing were proposed by Lodi and Monaci [123] for the 2D-KP and by Lodi, Martello, and Vigo [121] for the 2D-BPP and the 2D-SPP. These models were later extended to the 3-staged case of the 2D-BPP by Puchinger and Raidl [154] and to the 2-staged 2D-CSP with variable-sized bins by Furini and Malaguti [80].

\subsection{Pseudo-polynomial Models}

While polynomial models associate variables to the items, pseudo-polynomial models include variables associated with the positions into the bins where the items can be packed.

Beasley [13] proposed an ILP model for the 2D-KP, based on a discretization of the packing area through a pseudo-polynomial number of two-dimensional coordinates (see Section 3.1) where the bottom-left corner of an item can be packed. A pseudo-polynomial number of constraints imposes that no unit square is covered by more than one item. This formulation was later used for the solution of other two-dimensional packing problems. Alvarez-Valdes, Parreño, and Tamarit [2] adapted the formulation to the special case of the two-dimensional rectangular packing problem (see Section 2.1) with orthogonal rotations in which all items are identical (Pallet Loading Problem). de Queiroz and Miyazawa [156] adapted the model to a variant of the 2D-SPP in which the items have to be arranged to form a physically stable packing satisfying a predefined item unloading order. Martello and Monaci [127] used a similar model to solve the problems of orthogonally packing a set of rectangles (with or without allowing rotations) into the smallest square.

Valério de Carvalho [172] presented an arc-flow model for the solution of the 1D-CSP. The model is based on a digraph with a pseudo-polynomial number of vertices, in which paths correspond to feasible packings of a bin and a flow provides an overall problem solution. This formulation was 
extended to the 2-staged guillotine 2D-CSP by Macedo, Alves, and Valério de Carvalho [124] and to the 2-staged guillotine 2D-SPP by Mrad [142.

Another pseudo-polynomial formulation of the 1D-CSP that was further extended to twodimensional problems is the one-cut model, independently obtained by Rao [157] and Dyckhoff [70. The model is based on variables that describe the feasible cuts that split a bin (or a residual of a bin) into an item and a residual (or another item). Silva, Alvelos, and Valério de Carvalho [166] extended this model to the 2-staged and the 3-staged guillotine 2D-CSP, while Furini, Malaguti, and Thomopulos [82] used it for the non-staged guillotine 2D-SPP, 2D-CSP, and 2D-KP.

Another family of pseudo-polynomial models derives from time representations of scheduling problems where disjunctive constraints determine the relative positions between each pair of items. Following this methodology, Castro and Oliveira [39] developed ILP models for the 2D-SPP, and showed how to extend them to the 2D-BPP and to the 2D-KP. Castro and Grossmann [38] proposed further improvements for the 2D-SPP.

\subsection{Exponential Models}

Most exponential models for two-dimensional packing problems are based either on formulations that associate variables with feasible patterns (see Section 4.3), which have an exponential number of variables (columns), or on Benders' decomposition (see below), which produces an exponential number of constraints (rows). The models of the former type can be either set covering/set partitioning models (for bin packing or cutting stock problems) or set packing models (for knapsack problems).

\section{Branch(-and-cut)-and-price}

Models based on the set covering formulation are usually implemented through column generation (see Section 4.3) for solving the associated linear program relaxation. Embedding the linear program into an enumerative scheme allows one to obtain an optimal integer solution through branch-andprice or branch-and-cut-and-price (when cuts are added at the decision nodes).

To the best of our knowledge, Belov and Scheithauer [19] were the first to propose a branchand-cut-and-price algorithm ( based on a set-packing formulation) for a two-dimensional problem, namely the 2-staged guillotine 2D-KP.

Puchinger and Raidl [154] solved the 3-staged guillotine 2D-BPP with a branch-and-price algorithm with special techniques (column generation stabilization) to accelerate the convergence of the method, as previously suggested by Valério de Carvalho [174] and Ben Amor, Desrosiers, and Valério de Carvalho [7].

Pisinger and Sigurd [151, 152] proposed branch-and-price algorithms for 2D-BPPs with variablesized and fixed-sized bins. In these algorithms (which are, from a computational point of view, the current state-of-the-art), the columns are generated through decomposition into a one-dimensional knapsack problem and a 2D-OPP, which are then solved by constraint programming. Bettinelli, Ceselli, and Righini [25] developed a branch-and-price approach to the level (2-staged) 2D-SPP in which the pricing problem consists of a penalized one-dimensional knapsack problem. Furini and Malaguti [80] and Mrad, Meftahi and Haouari [143] solved the 2-staged guillotine 2D-CSP, respectively with and without variable-sized bins, through branch-and-price algorithms.

\section{Benders' Decomposition}

Already in 1962, Benders [20] proposed a method to decompose an MILP model having a special block structure into an MILP master problem and an LP slave problem. At each iteration, the slave 
receives the current master solution and either proves its optimality or generates a cut (Benders' cut) that is added to the master. The approach is also referred to as row generation.

Geoffrion [87] generalized the Benders' decomposition to the case in which the subproblem too is an MILP. The latter decomposition was used by Caprara and Monaci [36 and by Baldacci and Boschetti [10] to solve the 2D-KP: in both approaches the master is an ILP based on the one-dimensional knapsack problem while the slave solves an associated 2D-OPP.

Hooker and Ottosson [97] defined the logic-based Benders' decomposition for general MILP problems: in this approach both the master and the slave are MILPs, but the latter is solved through logical deduction methods, such as constraint programming. This decomposition was successfully applied by Pisinger and Sigurd [151, 152] to the 2D-KP and by de Queiroz et al. [155] to a special 2D-KP that includes pairs of items that cannot both be in the solution (2D-KP with conflict graphs).

Although Benders' decomposition has been successfully applied to cutting and packing problems, the resulting cuts in the master problem may be weak. Codato and Fischetti [53] defined stronger combinatorial Benders' cuts for general integer problems. Côté, Dell'Amico, and Iori [56] and Delorme, Iori, and Martello [66] adopted such cuts for the solution of the 2D-SPP without and with item rotation, respectively. In their logic-based Benders' decomposition, the master problem is a parallel processor scheduling problem with contiguity constraints (see Section 4.5) that produces the $x$-coordinate of each item, while the slave checks whether feasible $y$-coordinates exist. In the negative case, heuristic methods look for a minimal infeasible set of items that produces a combinatorial Benders' cut. A similar decomposition, with standard Benders' cuts, was used by Côté, Gendreau, and Potvin [57] for the 2D-OPP with unloading constraints.

Recently, Martin et al. 132 proposed a Benders' decomposition algorithm to solve the guillotine 2D-KP. In their decomposition, the master problem is modeled using the pseudo-polynomial model by Beasley [13], whereas the slave checks the guillotine restriction.

\section{Exact Methods based on Implicit Enumeration}

The algorithms reviewed in this section consist of enumeration schemes that do not explicitly make use of MILP models and hence do not require the use of a solver.

\subsection{Branch-and-Bound}

Several enumeration algorithms were derived from the bottom-left strategy proposed by Baker, Coffman, and Rivest [9] (see Section 5.2), which produces an approximate solution by placing one item at a time, in the lowest possible feasible position, left justified. Hadjiconstantinou and Christofides [91] developed a tree-search exact algorithm for the 2D-KP that packs the next item in every possible position such that the item's left and bottom edges touch either the bin or edges of other items (left-most downward strategy). Martello and Vigo [131] adopted this strategy in a branch-and-bound algorithm for the 2D-OPP, and embedded it in a two-level enumeration algorithm for the 2D-BPP.

This strategy later evolved into two classes of implicit enumeration schemes, namely the staircase placement and the niche placement. Both strategies enumerate the possible packing of the items with their bottom-left corner in particular positions (corner points), induced by the current (partial) packing, whose definition depends on the specific strategy. 


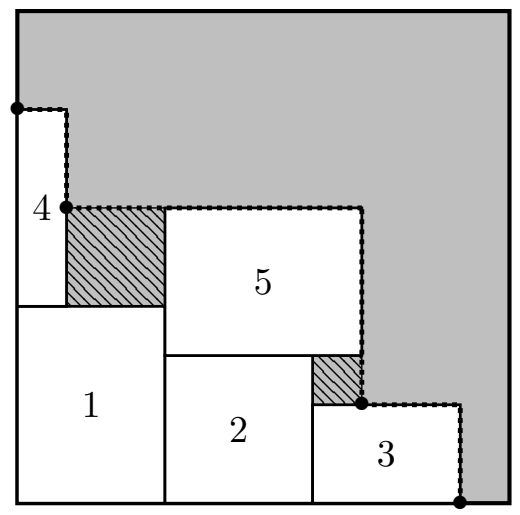

(a)

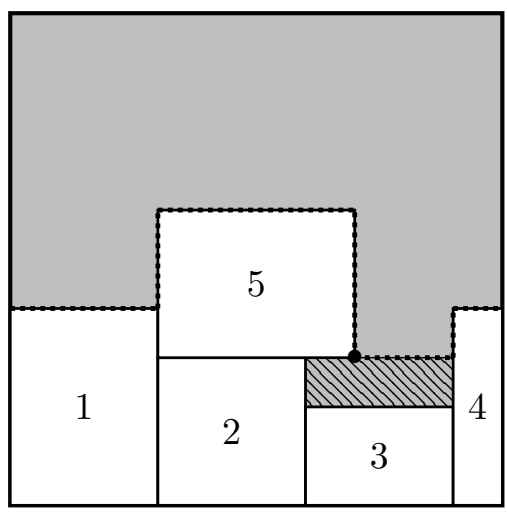

(b)

Figure 5: corner points of (a) staircase placement; (b) niche placement.

\section{Staircase Placement}

The staircase placement makes use of the envelope (see Figure 5 (a)), defined as the monotone (right-down) staircase-like boundary, that: (i) separates the area where previously packed items are placed from the area available for the remaining items, and (ii) is composed by segments touching at least one edge of a packed item or the bin. The corner points are those in which the slope of the envelope changes from vertical to horizontal. These concepts were originally introduced by Scheithauer [163.

Martello, Monaci, and Vigo [128] proposed a branch-and-bound algorithm for the 2D-SPP which is based on the staircase placement and on a strategy that avoids the enumeration of symmetric solutions. The method was later modified by Iori, Salazar González, and Vigo [103] to solve the 2D-OPP with loading/unloading constraints. Other branch-and-bound algorithms based on the staircase placement were proposed by Alvarez-Valdes, Parreño, and Tamarit [5] for the 2D-SPP, by Clautiaux, Carlier, and Moukrim [48] for the 2D-OPP, and by Bekrar et al. [15] for the 2D-SPP with guillotine constraint. The latter algorithm makes use of a method by Messaoud, Chu, and Espinouse [136] to detect non-guillotine patterns in polynomial time.

Recently, $\mathrm{Xu}$ and Lee [181] studied the continuous berth allocation problem, in which incoming vessels need to be assigned a time and a berth location on a quay. Each vessel can be interpreted as a rectangle with given width (the space it occupies in the berth) and height (the time it consumes): the problem is to "pack" the vessels into a strip of given width (the total space available at the berth) so as to minimize the total weighted completion times of the activities on the vessels. They solved the problem with a branch-and-bound algorithm that uses a staircase branching rule and obtains at each node a valid lower bound by invoking a model reformulation.

\section{Niche Placement}

The niche placement uses the skyline structure (see Figure 5(b)), a boundary composed by orthogonal line segments separating the area of previously packed items from the area that is available for the remaining items. At each iteration, the niche is the lowest and (in case of ties) leftmost horizontal segment: the left extreme of the niche is the only corner point considered for packing the next item.

A complete branching is obtained by creating at most $n+1$ nodes as follows. The first $n$ (at most) nodes are created by packing an item in the niche (obviously disregarding those that would 
lead to overlapping). Then, a last node in which no item is packed in the niche is also created. In such node, the niche is closed, so that at the next iteration a new niche will be considered.

The niche placement was used by Boschetti and Montaletti 32 in a branch-and-bound algorithm for the 2D-SPP and by Lesh et al. [115] for the 2D-SPP with perfect packing (a packing in which no wasted space is allowed). Kenmochi et al. [110] proposed two branch-and-bound algorithms, respectively adopting niche and staircase placement, for the 2D-SPP with perfect packing, with or without orthogonal rotation. They additionally derived algorithms for the general 2D-SPP. Improvements of the latter algorithms were presented by Arahori, Imamichi, and Nagamochi [8].

\section{Other Enumeration Schemes}

Certain solution methods for the 2D-KP are based on an enumeration of candidate subsets of items combined with an inner solution method that checks whether the current subset can be feasibly packed. This method has been used by Caprara and Monaci [36] for the 2D-KP and by Fekete, Schepers, and van der Veen 75 for knapsack problems with an arbitrary number of dimensions.

Christofides and Whitlock [45] and Christofides and Hadjiconstantinou [43] introduced enumerative approaches for the 2D-KP with guillotine constraints. Their algorithms are based on a tree-search approach where branchings correspond to cuts on a rectangle and bounds are obtained by solving relaxations through dynamic programming. Dolatabadi, Lodi, and Monaci 68] improved this enumeration scheme, obtaining a branch-and-bound and a branch-and-cut algorithm. Another search strategy that has been extensively used for problems with guillotine constraints is the bottom-up approach (see, e.g., Viswanathan and Bagchi [177], Hifi [96], Cung, Hifi, and Le Cun [62], and Fleszar [77]). It is based on enumerating all feasible patterns obtained through builds: a build is a rectangle produced by the (horizontal or vertical) combination of a pair of items or other builds. The bottom-up approach also inspired the development of MILP formulations for guillotine problems (see, e.g., Martin, Morabito, and Munari [133]).

A special case of 2-staged guillotine cutting patterns are the checkerboard patterns. While the guillotine constraint allows each cut to separate a bin into two pieces, which can then be treated as new (smaller) bins, the checkerboard constraint imposes that the solution be obtained through a set of horizontal cuts and a set of vertical cuts, all performed on the original bin. Yanasse and Katsurayama [182, 183] presented enumerative algorithms for the 2D-KP with checkerboard patterns.

Clautiaux et al. [52] proposed label setting algorithms for the four-staged guillotine 2D-KP. They presented reduction procedures, filtering rules based on a Lagrangian relaxation, and a state space relaxation.

\subsection{Graph-Based Approaches}

Morabito and Arenales [140] proposed an exact algorithm for the guillotine 2D-KP based on AND/OR graphs. In an AND/OR-graph representation, the nodes correspond to rectangles, the arcs correspond to cuts, and cutting patterns are represented as complete paths in the graph.

Fekete and Schepers [73] proposed a graph-theoretical characterization of the packing of a set of items into a bin. Their representation makes use of two interval graphs, associated with the horizontal and vertical dimensions, respectively: each item corresponds to a vertex, and two vertices are connected by an edge if and only if the projections of the corresponding items on the horizontal/vertical axis overlap. Theoretical properties allow one to detect the feasibility of a packing. This interval graph model easily extends to packings in higher dimensions.

Based on the interval graph representation, Fekete, Schepers, and van der Veen [75] proposed a branch-and-bound algorithm for higher-dimensional orthogonal packing problems. The algorithm 
was later improved by Belov and Rohling [18 through LP bounds based on the bar relaxation (see Section 4.5). In order to avoid the enumeration of symmetrical interval graphs and unnecessarily enumerated packing classes (as observed by Ferreira and Oliveira [76]), Joncour and Pêcher [107] proposed to adopt the so-called consecutive ones matrices to enumerate relevant interval graphs. Further improvements for the 2-dimensional case were proposed by Joncour, Pêcher, and Valicov 108.

Clautiaux, Jougler, and Moukrim [51] presented a graph-theoretical model for the 2D-OPP with guillotine constraints. The model is based on an arc-colored directed graph, which can be replaced by an uncolored undirected multigraph, and can be used for solving the problem through constraint programming.

\subsection{Constraint Programming}

As previously mentioned (see Section 6.3), Pisinger and Sigurd [151, 152] used a constraint programming formulation to solve 2D-OPP instances arising as subproblems in their decomposition approaches for the 2D-BPP (with fixed-sized or variable-sized bins). The main constraints are related to the relative positioning of each pair of items. This strategy was later adopted for other two-dimensional packing problems, e.g., by Korf, Moffitt, and Pollack [112] for the problem of orthogonally packing a set of two-dimensional items into a rectangle having minimum area, and by de Queiroz et al. [155] for a variant of the 2D-KP (see Section 6.3).

Clautiaux et al. 50] proposed a constraint-based scheduling model for the 2D-OPP, and solved it through constraint programming and effective propagation techniques. The approach was improved by Mesyagutov, Scheithauer, and Belov [137] in their algorithms for the 2D-SPP and the 2D-OPP, by embedding LP-based pruning rules into the constraint propagation process.

Soh et al. [169] solved the 2D-OPP by iteratively reducing it to satisfiability testing (SAT) problems, which look for a feasible assignment of a set of boolean variables. The approach extends to the solution of the 2D-SPP. Grandcolas and Pinto [90] proposed a SAT encoding of the interval graph model by Fekete and Schepers [73] for higher dimensional problems, and compared its efficiency with that of other SAT encodings.

Delorme, Iori, and Martello [66] proposed, for the 2D-CSP, a constraint programming formulation based on non-overlapping intervals to determine whether a solution of the contiguous one-dimensional relaxation (see Section 4.5) produces a feasible two-dimensional packing.

\section{Open Problems}

Our study shows that considerable improvements in the exact solution of two-dimensional cutting and packing problems emerged in the last decades, typically allowing to determine an optimal solution even for large-size instances. However, the inherent hardness of this class of problems is witnessed by the existence of unsolved instances with relatively few items. In the following, we provide a list of the main benchmarks that are still unsolved to proven optimality, not only for the main problems addressed in this survey, but also for other relevant problem variants:

- for the 2D-SPP, specific instances with 20 items cannot be solved to proven optimality even by recent and sophisticated algorithms (see, e.g., Côté, Dell'Amico, and Iori [56]). Among the 500 instances of the so called 10 classes benchmark (proposed a long time ago by Berkey and Wang [24] and Martello and Vigo [131] for the 2D-BPP), only 322 are solved to proven optimality. A number of instances with 40 items are still open for the 2D-SPP with guillotine cuts (see, e.g., Mrad [142]); 
- the 2D-SPP with item rotation is even more difficult from a computational perspective: among the above mentioned 500 instances, just 176 could be solved to optimality, and in particular 56 instances with only 20 items are still open (see, e.g., Delorme, Iori, and Martello [66]);

- the work by Delorme, Iori, and Martello [66] also presents extensive computational results for the pallet loading problem (a 2D-OPP variant in which all items have the same dimensions and rotation of 90 degrees is allowed) and the rectangle packing problem (a 2D-SPP variant in which items must be packed, without rotation, in a square of minimum area, see Martello and Monaci [127]). For the former problem, a few dozen instances remain unsolved (see http://lagrange.ime.usp.br/ lobato/packing/cover3.php for an up-to-date list). For the latter problem, more than 200 instances are unsolved, 50 of these (set RND_R15 in [127]) involving just 15 items;

- after more than 20 years, many 2D-BPP instances of the 10 classes benchmark (see [24], [131]) with $n \geq 60$ are still unsolved, and the same holds even for some instances with $n=40$ (see Pisinger and Sigurd [152]). For all such instances the difference between the best upper and lower bounds is one bin;

- Pisinger and Sigurd 151 created 500 instances of the 2D-BPP with variable bin sizes and costs, by modifying the 10 classes above. They could only solve 164 of them, leaving as open problems 29 instances with 20 items, and 62 with 40 items. All instances are available at https://www . computational-logistics.org/orlib/topic/2D $\% 20$ Variable-sized $\% 20 \mathrm{Bin} \%$ 20Packing/index.html\#intro ;

- a very difficult 2D-KP instance with only 32 items, proposed 35 years ago by Beasley [13, is still open (see Caprara and Monaci [36] and Baldacci and Boschetti [10]). For the 2D-KP with guillotine constraints, several instances with either 25 or 50 items, recently proposed by Velasco and Uchoa [176], are open;

- for the 2D-OPP, two difficult sets of instances have been created by Mesyagutov, Scheithauer, and Belov [137], and can be downloaded at http://www.math.tu-dresden.de/ capad/ TESTS/OPP/12_cp2_data.zip. The zipfile includes, among others, 1080 instances (Gleb_lpcs and Gleb_opp_gen) with 20 items and bin width equal to either 100 or 1000, 18 of them being still unsolved. Other sets, known as $\mathrm{C}, \mathrm{N}$, and $\mathrm{T}$ (available at https://www.euro-online. org/websites/esicup/data-sets/\#1535972088188-55fb7640-4228), contain 91 instances with about 70 items and bin width at most 200: only 39 of them have been solved to optimality (see Côté and Iori [58]);

- for the 2D-OPP with unloading constraints, Côté, Gendreau, and Potvin [57] generated 6 classes containing in total 3282 instances, with 26 items per instance on average: only 2179 of them have been solved to proven optimality. The instances can be downloaded from https://w1.cirrelt.ca/ cotejean/ (see 2OPP-UL).

Most of the above instances can also be downloaded from Internet repositories like, e.g., the wellknown OR Library http://people.brunel.ac.uk/ mastjjb/jeb/info.html by John Beasley [14] or the library of the OR group of the University of Bologna http://or dei.unibo.it/library. In addition, a generator of instances for two-dimensional rectangular cutting and packing problems, proposed by Silva, Oliveira, and Wäscher [167], can be downloaded from https://sites.google. com/gcloud.fe.up.pt/cutting-and-packing-tools. 


\section{Conclusions and Future Research Directions}

We reviewed over 180 papers related to two-dimensional orthogonal cutting and packing problems. The literature in this field has grown considerably in recent years, as also shown by the fact that about half of our references appeared in the last ten years, and just $20 \%$ were published before 2000. We described the main preprocessing and relaxation methods and briefly examined heuristic and approximation algorithms. We discussed mathematical models and reviewed the most effective implicit enumeration approaches. Finally, we provided an extensive list of instances for which the optimal solution is still unknown.

To facilitate future research, we provide in Table 1 a summary of the publications from the last 20 years on exact methods for $2 \mathrm{D}$ problems. The table presents the main problems, variants and techniques studied by each paper. The problems are divided into the four main categories discussed in Section 2 (column BPP gives both references to 2D-BPP and 2D-CSP). The variants are given in the columns OR (orthogonal rotation), GL (guillotine cuts), VS (variable sized bins), LU (loading/unloading constraints) and OTH (other variants). The main techniques are shown in columns MILP, B\&B (branch-and-bound), B\&P (branch-(-and-cut)-and-price), BD (Benders' decomposition), GB (graph based), CP (constraint programming), and REL (relaxations).

Some statistics on the recent exact methods can be drawn from the references in the table: 20 papers studied the 2D-SPP, 17 studied the 2D-BPP and/or the 2D-CSP, 14 studied the 2D-KP, and 19 studied the 2D-OPP. The most studied variant is the guillotine case. The most popular solution methods are MILP and branch-and-bound (both with 14 references). Note, however, that the number of papers on Benders' decomposition is relatively high although the use of this technique for cutting and packing problems is quite recent.

We also provide the reader some hints of what we consider promising future research directions:

- Constraint Programming, either as a stand-alone algorithm or as a sub-problem in decomposition methods, recently led to consistent improvements in computational results on $2 \mathrm{D}$ problems. Further research on this type of methodology is envisaged, as we see the potential to obtain further improvements;

- the use of Benders' decompositions (with or without Constraint Programming) for solving 2D problems is quite recent. For some of the classical problems, these decompositions represent the state-of-the-art, as they could solve to proven optimality several open instances. Such techniques are easily adaptable to many other problem variants, as one can consider embedding the additional features either in the master or in the slave. We thus envisage further research on this decomposition to solve new problem variants;

- current research trends appear to be intensive for 2D problems with additional constraints, like loading/unloading, stability, fragility, etc.;

- most of the techniques we presented can be generalized to the case of three or more dimensions, although this is not always straightforward. The amount of research on three-dimensional cutting and packing problems has been so far quite limited, in spite of their large number of applications. Interesting research directions can be explored for these problems, starting from the $2 \mathrm{D}$ ones.

We hope that this review will encourage researchers to pursue investigations in these fascinating topics where there is still room for improving the algorithmic approaches. 
Table 1: Summary of papers with exact methods and relaxations from the last 20 years.

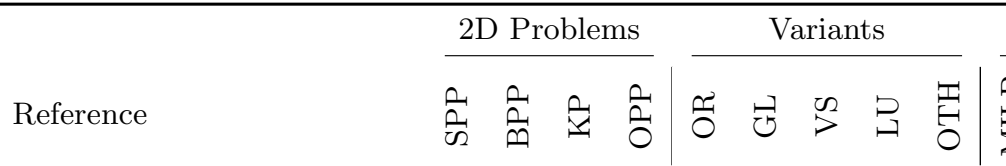

\begin{tabular}{|c|c|c|c|c|c|c|}
\hline \multicolumn{7}{|c|}{ Techniques } \\
\hline 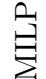 & $\begin{array}{l}\frac{n}{3} \\
m \\
m\end{array}$ & $\begin{array}{l}\frac{D}{D} \\
m\end{array}$ & $\hat{\oplus}$ & $\stackrel{\vartheta}{\mho}$ & $\theta$ & 空 \\
\hline
\end{tabular}

Alvarez-Valdes, Parreño, and Tamarit 5

Arahori, Imamichi, and Nagamochi 8

Baldacci and Boschetti 10

Bekrar et al. 15.

Belov et al. 16

Belov et al. 17.

Belov and Rohling 18

Belov and Scheithauer 19

Bettinelli, Ceselli, and Righini 25

Boschetti, Hadjiconstantinou, and Mingozzi 29]

Boschetti and Mingozzi 30

Boschetti and Mingozzi 31

Boschetti and Montaletti 32

Caprara and Monaci 36 .

Caprara and Monaci 37

Carlier, Clautiaux, and Moukrim 49

Castro and Grossmann 38

Castro and Oliveira 39

Cintra et al. 46.

Clautiaux, Carlier, and Moukrim 49

Clautiaux et al. [50]

Clautiaux, Jouglet, and Moukrim 51

Clautiaux et al. 52.

Côté, Dell'Amico, and Iori 56

Côté, Gendreau, and Potvin 57

Delorme, Iori, and Martello 66

Dolatabadi, Lodi, and Monaci 68.

Fekete and Schepers 73

Fekete and Schepers 74

Fekete, Schepers, and Veen 75

Fleszar 77 .

Friedow and Scheithauer 78

Furini and Malaguti 80

Furini, Malaguti, and Thomopulos 82

Grandcolas and Pinto 90

Iori, Salazar-Gonzalez, and Vigo 102

Joncour and Pêcher 107

Joncour, Pêcher, and Valicov [108.

Kenmochi et al. 110

Lesh et al. 115

Lodi, Martello, and Vigo 121

Lodi and Monaci 123

Macedo, Alves, and Valério de Carvalho 124

Martello, Monaci, and Vigo [128.

Martin et al. 132

Martin, Morabito, and Munari 133 .

Matayoshi 134

Melega, de Araujo, and Jans 135

Messaoud et al. 136

Mesyagutov, Scheithauer, and Belov 137

Mesyagutov et al. 138

Mrad 142

Pisinger and Sigurd 151

Pisinger and Sigurd 152

Puchinger and Raidl 154

Queiroz et al. 155

Queiroz and Miyazawa [156]

Serairi and Haouari [165]

Silva, Alvelos, and Valério de Carvalho [166.

Soh et al. 169

Velasco and Uchoa 176 .

Yanasse and Katsurayama 182

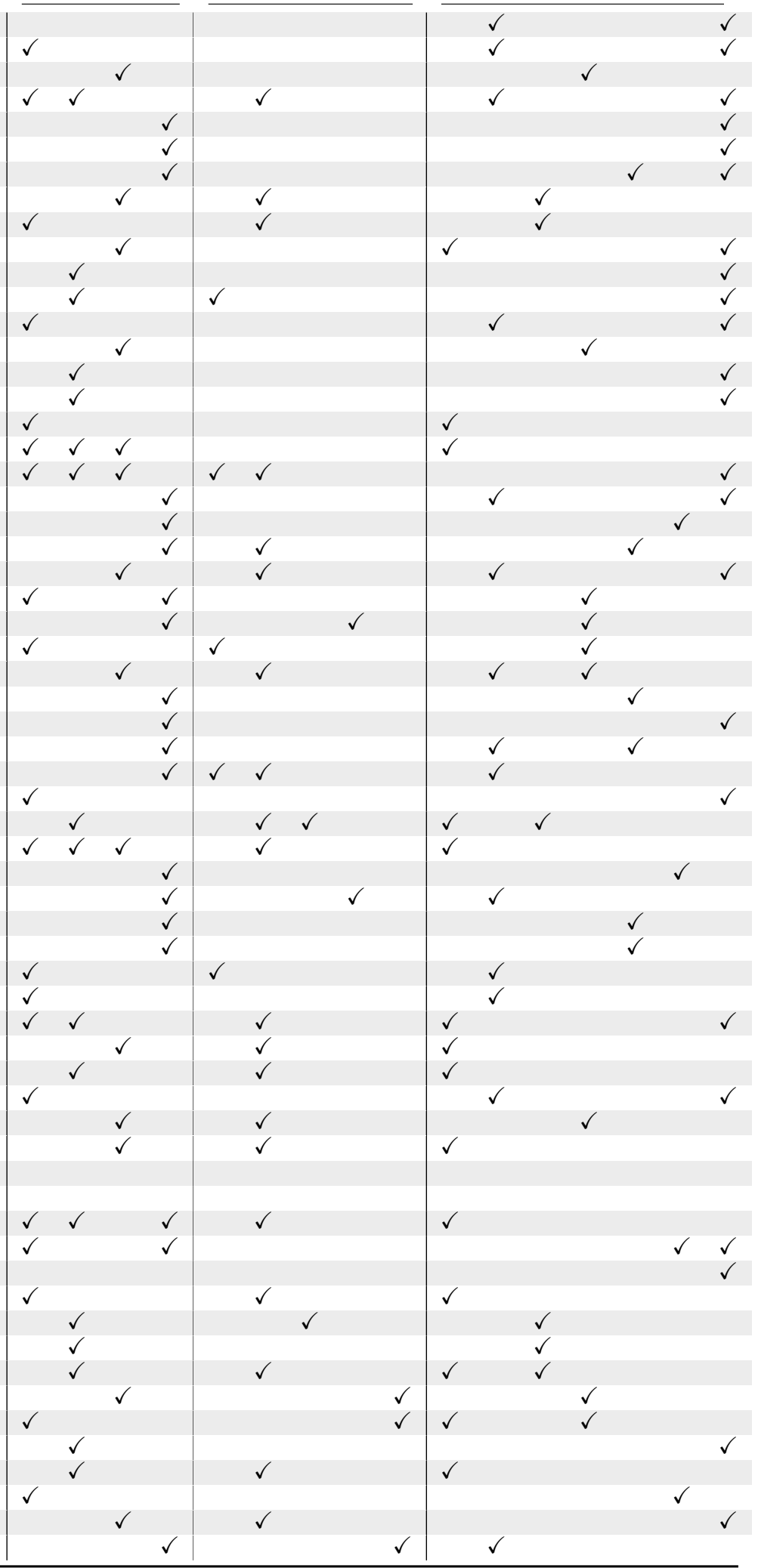




\section{Acknowledgments}

Research supported by Air Force Office of Scientific Research, by CNPq (under grant numbers 425340/2016-3 and 314366/2018-0), and by FAPESP (under grant numbers 2015/11937-9, 2016/018601, 2016/23552-7, 2018/19217-3, and 2019/12728-5).

\section{References}

[1] R. Alvarez-Valdés, A. Parajón, and J.M. Tamarit. A tabu search algorithm for large-scale guillotine (un)constrained two-dimensional cutting problems. Computers \& Operations Research, 29(7):925-947, 2002.

[2] R. Alvarez-Valdes, F. Parreño, and J.M. Tamarit. A branch-and-cut algorithm for the pallet loading problem. Computers \& Operations Research, 32(11):3007-3029, 2005.

[3] R. Alvarez-Valdes, F. Parreño, and J.M. Tamarit. A tabu search algorithm for a twodimensional non-guillotine cutting problem. European Journal of Operational Research, 183(3):1167-1182, 2007.

[4] R. Alvarez-Valdes, F. Parreño, and J.M. Tamarit. Reactive GRASP for the strip-packing problem. Computers \& Operations Research, 35(4):1065-1083, 2008.

[5] R. Alvarez-Valdes, F. Parreño, and J.M. Tamarit. A branch and bound algorithm for the strip packing problem. OR Spectrum, 31(2):431-459, 2009.

[6] C. Alves, F. Clautiaux, J.M. Valério de Carvalho, and J. Rietz. Dual-Feasible Functions for Integer Programming and Combinatorial Optimization. Springer International Publishing, Cham, 2016.

[7] H. Ben Amor, J. Desrosiers, and J.M. Valério de Carvalho. Dual-optimal inequalities for stabilized column generation. Operations Research, 54(3):454-463, 2006.

[8] Y. Arahori, T. Imamichi, and H. Nagamochi. An exact strip packing algorithm based on canonical forms. Computers \& Operations Research, 39(12):2991-3011, 2012.

[9] B.S. Baker, E.G. Coffman, Jr., and R.L. Rivest. Orthogonal packing in two dimensions. SIAM Journal on Computing, 9(4):846-855, 1980.

[10] R. Baldacci and M.A. Boschetti. A cutting-plane approach for the two-dimensional orthogonal non-guillotine cutting problem. European Journal of Operational Research, 183(3):1136-1149, 2007.

[11] J. Balogh, J. Békési, G. Dósa, L. Epstein, and A. Levin. Lower bounds for several online variants of bin packing. In Approximation and Online Algorithms - 15th International Workshop, WAOA 2017, Revised Selected Papers, pages 102-117. Springer Verlag, 2018.

[12] J.E. Beasley. Algorithms for unconstrained two-dimensional guillotine cutting. Journal of the Operational Research Society, 36(4):297-306, 1985.

[13] J.E. Beasley. An exact two-dimensional non-guillotine cutting tree search procedure. Operations Research, 33(1), 1985. 
[14] J.E. Beasley. Or-library: distributing test problems by electronic mail. Journal of the Operational Research Society, 41(11):1069-1072, 1990.

[15] A. Bekrar, I. Kacem, C. Chu, and C. Sadfi. An improved heuristic and an exact algorithm for the 2D strip and bin packing problem. International Journal of Product Development, 10(1-3):217-240, 2010.

[16] G. Belov, V. M. Kartak, H. Rohling, and G. Scheithauer. One-dimensional relaxations and LP bounds for orthogonal packing. ITOR, 16(6):745-766, 2009.

[17] G. Belov, V. M. Kartak, H. Rohling, and G. Scheithauer. Conservative scales in packing problems. OR Spectrum, 35(2):505-542, 2013.

[18] G. Belov and H. Rohling. LP bounds in an interval-graph algorithm for orthogonal-packing feasibility. Operations Research, 61(2):483-497, 2013.

[19] G. Belov and G. Scheithauer. A branch-and-cut-and-price algorithm for one-dimensional stock cutting and two-dimensional two-stage cutting. European Journal of Operational Research, 171(1):85-106, 2006.

[20] J.F. Benders. Partitioning procedures for solving mixed variables programming problems. Numerische Mathematik, 4(1):238-252, 1962.

[21] J. A. Bennell, J.F. Oliveira, and G. Wäscher. Cutting and packing. International Journal of Production Economics, 145(2):449-450, 2013.

[22] J.A. Bennell and J.F. Oliveira. The geometry of nesting problems: A tutorial. European Journal of Operational Research, 184(2):397-415, 2008.

[23] J.A. Bennell and J.F. Oliveira. A tutorial in irregular shape packing problems. Journal of the Operational Research Society, 60(supp 1):S93-S105, 2009.

[24] J. O. Berkey and P. Y. Wang. Two dimensional finite bin packing algorithms. Journal of the Operational Research Society, 38:423-429, 1987.

[25] A. Bettinelli, A. Ceselli, and G. Righini. A branch-and-price algorithm for the twodimensional level strip packing problem. 4OR, 6(4):361-374, 2008.

[26] V.M.R. Bezerra, A.A.S. Leao, J.F. Oliveira, and M.O. Santos. Models for the two-dimensional level strip packing problem - a review and a computational evaluation. Journal of the Operational Research Society, 71(4):606-627, 2020.

[27] I. Borgulya. A parallel hyper-heuristic approach for the two-dimensional rectangular strippacking problem. Journal of computing and information technology, 22(4):251-265, 2014.

[28] A. Bortfeldt and G. Wäscher. Constraints in container loading - A state-of-the-art review. European Journal of Operational Research, 229(1):1-20, 2013.

[29] M.A. Boschetti, E. Hadjiconstantinou, and A. Mingozzi. New upper bounds for the twodimensional othogonal non guillotine cutting stock problem. IMA Journal of Management Mathematics, 13(2):95-119, 2002.

[30] M.A. Boschetti and A. Mingozzi. The two-dimensional finite bin packing problem. Part I: New lower bounds for the oriented case. 4OR, 1(1):27-42, 2003. 
[31] M.A. Boschetti and A. Mingozzi. The two-dimensional finite bin packing problem. Part II: New lower and upper bounds. 4OR, 1(2):135-147, 2003.

[32] M.A. Boschetti and L. Montaletti. An exact algorithm for the two-dimensional strip-packing problem. Operations Research, 58(6):1774-1791, 2010.

[33] E. K. Burke, M. Hyde, G. Kendall, and J. Woodward. A genetic programming hyper-heuristic approach for evolving 2-D strip packing heuristics. IEEE Transactions on Evolutionary Computation, 14(6):942-958, 2010.

[34] E.K. Burke, M.R. Hyde, and G. Kendall. A squeaky wheel optimisation methodology for two-dimensional strip packing. Computers \& Operations Research, 38(7):1035-1044, 2011.

[35] E.K. Burke, G. Kendall, and G. Whitwell. A new placement heuristic for the orthogonal stock-cutting problem. Operations Research, 52(4):655-671, 2004.

[36] A. Caprara and M. Monaci. On the two-dimensional knapsack problem. Operations Research Letters, 32(1):5-14, 2004.

[37] A. Caprara and M. Monaci. Bidimensional packing by bilinear programming. Mathematical Programming, 118(1):75-108, 2009.

[38] P.M. Castro and I.E. Grossmann. From time representation in scheduling to the solution of strip packing problems. Computers \& Chemical Engineering, 44:45-57, 2012.

[39] P.M. Castro and J.F. Oliveira. Scheduling inspired models for two-dimensional packing problems. European Journal of Operational Research, 215(1):45-56, 2011.

[40] B. Chazelle. The bottomn-left bin-packing heuristic: An efficient implementation. IEEE Transactions on Computers, C-32(8):697-707, 1983.

[41] C.S. Chen, S.M. Lee, and Q.S. Shen. An analytical model for the container loading problem. European Journal of Operational Research, 80(1):68-76, 1995.

[42] H. I. Christensen, A. Khan, S. Pokutta, and P. Tetali. Approximation and online algorithms for multidimensional bin packing: A survey. Computer Science Review, 24:63-79, 2017.

[43] N. Christofides and E. Hadjiconstantinou. An exact algorithm for orthogonal 2-d cutting problems using guillotine cuts. European Journal of Operational Research, 83(1):21-38, 1995.

[44] N. Christofides, A. Mingozzi, and P. Toth. State-space relaxation procedures for the computation of bounds to routing problems. Networks, 11(2):145-164, 1981.

[45] N. Christofides and C. Whitlock. An algorithm for two-dimensional cutting problems. Oper. Res., 25(1):30-44, 1977.

[46] G.F. Cintra, F.K. Miyazawa, Y. Wakabayashi, and E.C. Xavier. Algorithms for twodimensional cutting stock and strip packing problems using dynamic programming and column generation. European Journal of Operational Research, 191(1):61-85, 2008.

[47] F. Clautiaux, C. Alves, and J.M. Valério de Carvalho. A survey of dual-feasible and superadditive functions. Annals of Operations Research, 179(1):317-342, 2010. 
[48] F. Clautiaux, J. Carlier, and A. Moukrim. A new exact method for the two-dimensional orthogonal packing problem. European Journal of Operational Research, 183(3):1196-1211, 2007.

[49] F. Clautiaux, J. Carlier, and A. Moukrim. New reduction procedures and lower bounds for the two-dimensional bin packing problem with fixed orientation. Computers 83 Operations Research, 34(8):2223-2250, 2007.

[50] F. Clautiaux, A. Jouglet, J. Carlier, and A. Moukrim. A new constraint programming approach for the orthogonal packing problem. Computers $\&$ Operations Research, 35(3):944-959, 2008 .

[51] F. Clautiaux, A. Jouglet, and A. Moukrim. A new graph-theoretical model for $k$-dimensional guillotine-cutting problems. In Catherine C. McGeoch, editor, Experimental Algorithms, pages 43-54, Berlin, Heidelberg, 2008. Springer Berlin Heidelberg.

[52] F. Clautiaux, R. Sadykov, F. Vanderbeck, and Q. Viaud. Combining dynamic programming with filtering to solve a four-stage two-dimensional guillotine-cut bounded knapsack problem. Discrete Optimization, 29:18-44, 2018.

[53] G. Codato and M. Fischetti. Combinatorial Benders' cuts for mixed-integer linear programming. Operations Research, 54(4):756-766, 2006.

[54] E.G. Coffman Jr., J. Csirik, G. Galambos, S. Martello, and D. Vigo. Bin Packing Approximation Algorithms: Survey and Classification, pages 455-531. Springer New York, New York, NY, 2013.

[55] G. Costa, M. Delorme, M. Iori, E. Malaguti, and S. Martello. Training software for orthogonal packing problems. Computers \& Industrial Engineering, 111:139-147, 2017.

[56] J.-F. Côté, M. Dell'Amico, and M. Iori. Combinatorial Benders' cuts for the strip packing problem. Operations Research, 62(3):643-661, 2014.

[57] J.-F. Côté, M. Gendreau, and J.-Y. Potvin. An exact algorithm for the two-dimensional orthogonal packing problem with unloading constraints. Operations Research, 62(5):11261141, 2014.

[58] J.-F Côté and M. Iori. The meet-in-the-middle principle for cutting and packing problems. INFORMS Journal on Computing, 30(4):646-661, 2018.

[59] T.G. Crainic, G. Perboli, and R. Tadei. Recent advances in multi-dimensional packing problems. In Constantin Volosencu, editor, New Technologies - Trends, Innovations and Research. IntechOpen, Rijeka, 2012.

[60] Y. Cui and Z. Zhao. Heuristic for the rectangular two-dimensional single stock size cutting stock problem with two-staged patterns. European Journal of Operational Research, 231(2):288-298, 2013.

[61] Y.-P. Cui, Y. Zhou, and Y. Cui. Triple-solution approach for the strip packing problem with two-staged patterns. Journal of Combinatorial Optimization, 34(2):588-604, 2017.

[62] V.-D. Cung, M. Hifi, and B. Le Cun. Constrained two-dimensional cutting stock problems a best-first branch-and-bound algorithm. International Transactions in Operational Research, 7(3):185-210, 2000. 
[63] G.B. Dantzig. Discrete variable extremum problems. Operations Research, 5(2):266-277, 1957.

[64] M. Dell'Amico and S. Martello. Optimal scheduling of tasks on identical parallel processors. ORSA Journal on Computing, 7(2):191-200, 1995.

[65] M. Delorme, M. Iori, and S. Martello. Bin packing and cutting stock problems: Mathematical models and exact algorithms. European Journal of Operational Research, 255(1):1-20, 2016.

[66] M. Delorme, M. Iori, and S. Martello. Logic based Benders' decomposition for orthogonal stock cutting problems. Computers \& Operations Research, 78:290-298, 2017.

[67] M. Delorme, M. Iori, and S. Martello. BPPLIB: a library for bin packing and cutting stock problems. Optimization Letters, 12(2):235-250, 2018.

[68] M. Dolatabadi, A. Lodi, and M. Monaci. Exact algorithms for the two-dimensional guillotine knapsack. Computers \& Operations Research, 39(1):48-53, 2012.

[69] K. A. Dowsland and W. B. Dowsland. Solution approaches to irregular nesting problems. European Journal of Operational Research, 84:506-521, 1995.

[70] H. Dyckhoff. A new linear programming approach to the cutting stock problem. Operations Research, 29(6):1092-1104, 1981.

[71] H. Dyckhoff. A typology of cutting and packing problems. European Journal of Operational Research, 44(2):145-159, 1990.

[72] S.P. Fekete and J. Schepers. New classes of fast lower bounds for bin packing problems. Mathematical Programming, 91(1):11-31, 2001.

[73] S.P. Fekete and J. Schepers. A combinatorial characterization of higher-dimensional orthogonal packing. Mathematics of Operations Research, 29(2):353-368, 2004.

[74] S.P. Fekete and J. Schepers. A general framework for bounds for higher-dimensional orthogonal packing problems. Mathematical Methods of Operations Research, 60(2):311-329, 2004 .

[75] S.P. Fekete, J. Schepers, and J.C. van der Veen. An exact algorithm for higher-dimensional orthogonal packing. Operations Research, 55(3):569-587, 2007.

[76] E.P. Ferreira and J.F. Oliveira. Fekete and Schepers' graph-based algorithm for the twodimensional orthogonal packing problem revisited. In Bortfeldt A., Homberger J., Kopfer H., Pankratz G., and Strangmeier R., editors, Intelligent Decision Support, pages 15-31. Gabler, Wiesbaden, 2008.

[77] K. Fleszar. An exact algorithm for the two-dimensional stage-unrestricted guillotine cutting/packing decision problem. INFORMS Journal on Computing, 28(4):703-720, 2016.

[78] I. Friedow and G. Scheithauer. Using contiguous 2d-feasible 1d cutting patterns for the 2d strip packing problem. In Operations Research Proceedings 2015, pages 71-77. Springer International Publishing, 2017.

[79] D.K. Friesen and M.A. Langston. Variable sized bin packing. SIAM journal on computing, 15(1):222-230, 1986. 
[80] F. Furini and E. Malaguti. Models for the two-dimensional two-stage cutting stock problem with multiple stock size. Computers \& Operations Research, 40(8):1953-1962, 2013.

[81] F. Furini, E. Malaguti, R.M. Durán, A. Persiani, and P. Toth. A column generation heuristic for the two-dimensional two-staged guillotine cutting stock problem with multiple stock size. European Journal of Operational Research, 218(1):251-260, 2012.

[82] F. Furini, E. Malaguti, and D. Thomopulos. Modeling two-dimensional guillotine cutting problems via integer programming. INFORMS Journal on Computing, 28(4):736-751, 2016.

[83] W. Gálvez, F. Grandoni, S. Heydrich, S. Ingala, A. Khan, and A. Wiese. Approximating geometric knapsack via l-packings. In 2017 IEEE 58th Annual Symposium on Foundations of Computer Science (FOCS), pages 260-271, 2017.

[84] M.R. Garey and D.S. Johnson. Computers and Intractability: A Guide to the Theory of NP-Completeness. W.H. Freeman and Company, San Francisco, 1979.

[85] M. Gendreau, M. Iori, G. Laporte, and S. Martello. A tabu search algorithm for a routing and container loading problem. Transportation Science, 40(3):342-350, 2006.

[86] M. Gendreau, M. Iori, G. Laporte, and S. Martello. A tabu search heuristic for the vehicle routing problem with two-dimensional loading constraints. Networks, 51(1):4-18, 2008.

[87] A.M. Geoffrion. Generalized Benders decomposition. Journal of Optimization Theory and Applications, 10(4):237-260, 1972.

[88] P. C. Gilmore and R. E. Gomory. Multistage cutting stock problems of two and more dimensions. Operations Research, 13(1):94-120, 1965.

[89] J.F. Gonçalves and M.G.C. Resende. A biased random key genetic algorithm for 2D and 3D bin packing problems. International Journal of Production Economics, 145(2):500-510, 2013.

[90] S. Grandcolas and C. Pinto. A sat encoding for multi-dimensional packing problems. In Andrea Lodi, Michela Milano, and Paolo Toth, editors, Integration of AI and OR Techniques in Constraint Programming for Combinatorial Optimization Problems, pages 141-146, Berlin, Heidelberg, 2010. Springer Berlin Heidelberg.

[91] E. Hadjiconstantinou and N. Christofides. An exact algorithm for general, orthogonal, twodimensional knapsack problems. European Journal of Operational Research, 83(1):39-56, 1995.

[92] E. Hadjiconstantinou and M. Iori. A hybrid genetic algorithm for the two-dimensional single large object placement problem. European Journal of Operational Research, 183(3):11501166, 2007.

[93] X. Han, K. Iwama, D. Ye, and G. Zhang. Approximate strip packing: Revisited. Information and Computation, 249:110-120, 2016.

[94] S. Henning, K. Jansen, M. Rau, and L. Schmarje. Complexity and inapproximability results for parallel task scheduling and strip packing. Theory of Computing Systems, 64:120-140, 2020.

[95] J.C. Herz. Recursive computational procedure for two-dimensional stock cutting. IBM Journal of Research and Development, 16(5):462-469, 1972. 
[96] M. Hifi. An improvement of Viswanathan and Bagchi's exact algorithm for constrained twodimensional cutting stock. Computers $\mathcal{E}$ Operations Research, 24(8):727-736, 1997.

[97] J.N. Hooker and G. Ottosson. Logic-based Benders decomposition. Mathematical Programming, 96(1):33-60, 2003.

[98] S. Imahori and M. Yagiura. The best-fit heuristic for the rectangular strip packing problem: An efficient implementation and the worst-case approximation ratio. Computers $\&$ Operations Research, 37(2):325-333, 2010.

[99] M. Iori and S. Martello. Routing problems with loading constraints. TOP, 18(1):4-27, 2010.

[100] M. Iori and S. Martello. An annotated bibliography of combined routing and loading problems. Yugoslav Journal of Operations Research, 23(3):311-326, 2013.

[101] M. Iori, S. Martello, and M. Monaci. Metaheuristic Algorithms for the Strip Packing Problem, pages 159-179. Springer US, 2003.

[102] M. Iori, J.J. Salazar González, and D. Vigo. An exact approach for the vehicle routing problem with two-dimensional loading constraints. Transportation Science, 41:253-264, 2007.

[103] M. Iori, J.-J. Salazar-Gonzlez, and D. Vigo. An exact approach for the vehicle routing problem with two-dimensional loading constraints. Transportation Science, 41(2):253-264, 2007.

[104] S. Jakobs. On genetic algorithms for the packing of polygons. European Journal of Operational Research, 88(1):165-181, 1996.

[105] K. Jansen and M. Rau. Closing the gap for pseudo-polynomial strip packing. 2019. (presented at ESA 2019, Munich). http://arxiv.org/abs/1712.04922.

[106] D. S. Johnson. Near-optimal bin packing algorithms. PhD thesis, MIT, Cambridge, MA, 1973.

[107] C. Joncour and A. Pêcher. Consecutive ones matrices for multi-dimensional orthogonal packing problems. Electronic Notes in Discrete Mathematics, 36:327-334, 2010.

[108] C. Joncour, A. Pêcher, and P. Valicov. MPQ-trees for the orthogonal packing problem. Electronic Notes in Discrete Mathematics, 11:423-429, 2012.

[109] H. Kellerer, U. Pferschy, and D. Pisinger. Knapsack Problems. Springer, Berlin, Germany, 2004.

[110] M. Kenmochi, T. Imamichi, K. Nonobe, M. Yagiura, and H. Nagamochi. Exact algorithms for the two-dimensional strip packing problem with and without rotations. European Journal of Operational Research, 198(1):73-83, 2009.

[111] I. Kierkosz and M. Luczak. A hybrid evolutionary algorithm for the two-dimensional packing problem. Central European Journal of Operations Research, 22(4):729-753, 2014.

[112] R.E. Korf, M.D. Moffitt, and M.E. Pollack. Optimal rectangle packing. Annals of Operations Research, 179(1):261-295, 2010.

[113] B. Kwon and G.M. Lee. Spatial scheduling for large assembly blocks in shipbuilding. Computers 85 Industrial Engineering, 89:203-212, 2015. 
[114] A.A.S. Leao, F.M.B. Toledo, J.F. Oliveira, M.A. Carravilla, and R. Alvarez-Valdés. Irregular packing problems: A review of mathematical models. European Journal of Operational Research, 282(3):803-822, 2020.

[115] N. Lesh, J. Marks, A. McMahon, and M. Mitzenmacher. Exhaustive approaches to 2D rectangular perfect packings. Information Processing Letters, 90(1):7-14, 2004.

[116] A. Lodi, S. Martello, and M. Monaci. Two-dimensional packing problems: A survey. European Journal of Operational Research, 141(2):241-252, 2002.

[117] A. Lodi, S. Martello, M. Monaci, C. Cicconetti, L. Lenzini, E. Mingozzi, C. Eklund, and J. Moilanen. Efficient two-dimensional packing algorithms for mobile WiMAX. Management Science, 57(12):2130-2144, 2011.

[118] A. Lodi, S. Martello, M. Monaci, and D. Vigo. Two-dimensional bin packing problems. In V. Th. Paschos, editor, Paradigms of Combinatorial Optimization: Problems and New Approaches, pages 107-129. John Wiley \& Sons, Ltd, Hoboken, NJ, USA, 2014.

[119] A. Lodi, S. Martello, and D. Vigo. Heuristic and metaheuristic approaches for a class of two-dimensional bin packing problems. INFORMS Journal on Computing, 11(4):345-357, 1999.

[120] A. Lodi, S. Martello, and D. Vigo. Recent advances on two-dimensional bin packing problems. Discrete Applied Mathematics, 123(1):379-396, 2002.

[121] A. Lodi, S. Martello, and D. Vigo. Models and bounds for two-dimensional level packing problems. Journal of Combinatorial Optimization, 8(3):363-379, 2004.

[122] A. Lodi, S. Martello, and D. Vigo. TSpack: a unified tabu search code for multi-dimensional bin packing problems. Annals of Operations Research, 131(1-4):203-213, 2004.

[123] A. Lodi and M. Monaci. Integer linear programming models for 2-staged two-dimensional knapsack problems. Mathematical Programming, 94(2):257-278, 2003.

[124] R. Macedo, C. Alves, and J.M. Valério de Carvalho. Arc-flow model for the two-dimensional guillotine cutting stock problem. Computers $\mathcal{E}$ Operations Research, 37(6):991-1001, 2010.

[125] S. Martello. Knapsack, packing and cutting. INFOR, 32(4):217-218, 1994.

[126] S. Martello. Two-dimensional packing problems in telecommunications. Pesquisa Operacional, $34(1): 31-38,2014$.

[127] S. Martello and M. Monaci. Models and algorithms for packing rectangles into the smallest square. Computers $\&$ Operations Research, 63:161-171, 2015.

[128] S. Martello, M. Monaci, and D. Vigo. An exact approach to the strip-packing problem. INFORMS Journal on Computing, 15(3):310-319, 2003.

[129] S. Martello and P. Toth. Lower bounds and reduction procedures for the bin packing problem. Discrete Applied Mathematics, 28(1):59-70, 1990.

[130] S. Martello and P. Toth. Knapsack Problems: Algorithms and Computer Implementations. John Wiley \& Sons, Chichester, 1990, (free download at http://www.or.deis.unibo.it/ knapsack.html). 
[131] S. Martello and D. Vigo. Exact solution of the two-dimensional finite bin packing problem. Management Science, 44(3):388-399, 1998.

[132] M. Martin, E.G. Birgin, R.D. Lobato, R. Morabito, and P. Munari. Models for the twodimensional rectangular single large placement problem with guillotine cuts and constrained pattern. International Transactions in Operational Research, 27(2):767-793, 2020.

[133] M. Martin, R. Morabito, and P. Munari. A bottom-up packing approach for modeling the constrained two-dimensional guillotine placement problem. Computers \& Operations Research, 115:104851, 2020.

[134] M. Matayoshi. The 2D strip packing problem: A new approach with verification by EA. In 2010 IEEE International Conference on Systems, Man and Cybernetics, pages 2492-2499, 2010.

[135] G.M. Melega, S.A. Araujo, and R. Jans. Classification and literature review of integrated lotsizing and cutting stock problems. European Journal of Operational Research, 271(1):1-19, 2018.

[136] S.B. Messaoud, C. Chu, and M.-L. Espinouse. Characterization and modelling of guillotine constraints. European Journal of Operational Research, 191(1):112 - 126, 2008.

[137] M. Mesyagutov, G. Scheithauer, and G. Belov. LP bounds in various constraint programming approaches for orthogonal packing. Computers $\&$ Operations Research, 39(10):2425-2438, 2012 .

[138] M.A. Mesyagutov, E.A. Mukhacheva, G.N. Belov, and Scheithauer G. Packing of onedimensional bins with contiguous selection of identical items: An exact method of optimal solution. Automation and Remote Control, 72(1):141-159, 2011.

[139] M. Monaci and P. Toth. A set-covering-based heuristic approach for bin-packing problems. INFORMS Journal on Computing, 18(1):71-85, 2006.

[140] R. Morabito and M.N. Arenales. Staged and constrained two-dimensional guillotine cutting problems: An AND/OR-graph approach. European Journal of Operational Research, 94(3):548-560, 1996.

[141] R. Morabito and V.A. Pureza. Heuristic approach based on dynamic programming and and/or-graph search for the constrained two-dimensional guillotine cutting problem. Annals of Operations Research, 179:297-315, 2010.

[142] M. Mrad. An arc flow-based optimization approach for the two-stage guillotine strip cutting problem. Journal of the Operational Research Society, 66(11):1850-1859, 2015.

[143] M. Mrad, I. Meftahi, and M. Haouari. A branch-and-price algorithm for the two-stage guillotine cutting stock problem. The Journal of the Operational Research Society, 64(5):629-637, 2013.

[144] V. Nesello, M. Delorme, M. Iori, and A. Subramanian. Mathematical models and decomposition algorithms for makespan minimization in plastic rolls production. Journal of the Operational Research Society, 69(3):326-339, 2018. 
[145] N. Ntene and J.H. van Vuuren. A survey and comparison of guillotine heuristics for the 2D oriented offline strip packing problem. Discrete Optimization, 6(2):174-188, 2009.

[146] J.F. Oliveira, A. Neuenfeldt Júnior, E. Silva, and M.A. Carravilla. A survey on heuristics for the two-dimensional rectangular strip packing problem. Pesquisa Operacional, 36(2):197-226, 2016 .

[147] J.F Oliveira and G. Wäscher. Cutting and packing. European Journal of Operational Research, 183(3):1106 - 1108, 2007.

[148] H. Onodera, Y. Taniguchi, and K. Tamaru. Branch-and-bound placement for building block layout. In 28th ACM/IEEE Design Automation Conference, pages 433-439, 1991.

[149] F.G. Ortmann and J.H. van Vuuren. Modified strip packing heuristics for the rectangular variable-sized bin packing problem. ORiON, 26(1):21-44, 2010.

[150] F. Parreño, R. Alvarez-Valdes, J.F. Oliveira, and J.M. Tamarit. A hybrid GRASP/VND algorithm for two- and three-dimensional bin packing. Annals of Operations Research, 179(1):203$220,2010$.

[151] D. Pisinger and M. Sigurd. The two-dimensional bin packing problem with variable bin sizes and costs. Discrete Optimization, 2(2):154-167, 2005.

[152] D. Pisinger and M. Sigurd. Using decomposition techniques and constraint programming for solving the two-dimensional bin-packing problem. INFORMS Journal on Computing, $19(1): 36-51,2007$.

[153] H. Pollaris, K. Braekers, A. Caris, G.K. Janssens, and S. Limbourg. Vehicle routing problems with loading constraints: State-of-the-art and future directions. OR Spectrum, 37(2):297-330, 2015 .

[154] J. Puchinger and G.R. Raidl. Models and algorithms for three-stage two-dimensional bin packing. European Journal of Operational Research, 183(3):1304-1327, 2007.

[155] T.A. de Queiroz, P.H.D.B. Hokama, R.C.S. Schouery, and F.K. Miyazawa. Two-dimensional disjunctively constrained knapsack problem: Heuristic and exact approaches. Computers $\&$ Industrial Engineering, 105:313-328, 2017.

[156] T.A. de Queiroz and F.K. Miyazawa. Order and static stability into the strip packing problem. Annals of Operations Research, 223(1):137-154, 2014.

[157] M.R. Rao. On the cutting stock problem. Journal of the Computer Society of India, 7:35-39, 1976.

[158] J. Rietz, C. Alves, and J.M. Valério de Carvalho. Theoretical investigations on maximal dual feasible functions. Operations Research Letters, 38(3):174-178, 2010.

[159] J. Rietz, C. Alves, and J.M. Valério de Carvalho. Worst-case analysis of maximal dual feasible functions. Optimization Letters, 6(8):1-19, 122011.

[160] J. Rietz, C. Alves, and J.M. Valério de Carvalho. On the extremality of maximal dual feasible functions. Operations Research Letters, 40(1):25-30, 2012. 
[161] M. Russo, M. Boccia, A. Sforza, and C. Sterle. Constrained two-dimensional guillotine cutting problem: upper-bound review and categorization. International Transactions in Operational Research, 27(2):794-834, 2020.

[162] M. Russo, A. Sforza, and C. Sterle. An exact dynamic programming algorithm for largescale unconstrained two-dimensional guillotine cutting problems. Computers $\&$ Operations Research, 50:97-114, 2014.

[163] G. Scheithauer. Equivalence and dominance for problems of optimal packing of rectangles. Ricerca Operativa, 83:3-34, 1997.

[164] G. Scheithauer. Introduction to Cutting and Packing Optimization. Springer International Publishing, 2018.

[165] M. Serairi and M. Haouari. A theoretical and experimental study of fast lower bounds for the two-dimensional bin packing problem. RAIRO-Operations Research, 52(2):391-414, 2018.

[166] E. Silva, F. Alvelos, and J.M. Valério de Carvalho. An integer programming model for twoand three-stage two-dimensional cutting stock problems. European Journal of Operational Research, 205(3):699-708, 2010.

[167] E. Silva, J.F. Oliveira, and G. Wäscher. 2DCPackGen: A problem generator for twodimensional rectangular cutting and packing problems. European Journal of Operational Research, 237(3):846-856, 2014.

[168] E. Silva, J.F. Oliveira, and G. Wäscher. The pallet loading problem: a review of solution methods and computational experiments. International Transactions in Operational Research, 23(1-2):147-172, 2016.

[169] T. Soh, K. Inoue, N. Tamura, M. Banbara, and H. Nabeshima. A sat-based method for solving the two-dimensional strip packing problem. Fundamenta Informaticae, 102(3-4):467487, 2010.

[170] T. Strecker and L. Hennig. Automatic layouting of personalized newspaper pages. In B. Fleischmann, K.-H. Borgwardt, R. Klein, and A. Tuma, editors, Operations Research Proceedings 2008, pages 469-474, Berlin, Heidelberg, 2009. Springer Berlin Heidelberg.

[171] J. Terno, R. Lindemann, and G. Scheithauer. Zuschnittprobleme and ihre praktische lösung. Technical report, Verlag Harry Deutsch, Thun und FrankfurtMain, 1987.

[172] J.M. Valério de Carvalho. Exact solution of bin-packing problems using column generation and branch-and-bound. Annals of Operations Research, 86(0):629-659, 1999.

[173] J.M. Valério de Carvalho. LP models for bin packing and cutting stock problems. European Journal of Operational Research, 141(2):253-273, 2002.

[174] J.M. Valério de Carvalho. Using extra dual cuts to accelerate column generation. INFORMS Journal on Computing, 17(2):175-182, 2005.

[175] F. Vanderbeck. A nested decomposition approach to a three-stage, two-dimensional cuttingstock problem. Management Science, 47(6):864-879, 2001.

[176] A.S. Velasco and E. Uchoa. Improved state space relaxation for constrained two-dimensional guillotine cutting problems. European Journal of Operational Research, 272(1):106-120, 2019. 
[177] K.V. Viswanathan and A. Bagchi. Best-first search methods for constrained two-dimensional cutting stock problems. Operations Research, 41(4):768-776, 1993.

[178] G. Wäscher, H. Haußner, and H. Schumann. An improved typology of cutting and packing problems. European Journal of Operational Research, 183(3):1109-1130, 2007.

[179] L. Wei, W.-C. Oon, W. Zhu, and A. Lim. A skyline heuristic for the 2D rectangular packing and strip packing problems. European Journal of Operational Research, 215(2):337-346, 2011.

[180] L. Wei, H. Qin, B. Cheang, and X. Xu. An efficient intelligent search algorithm for the twodimensional rectangular strip packing problem. International Transactions in Operational Research, 23(1-2):65-92, 2016.

[181] Z. Xu and C.-Y. Lee. New lower bound and exact method for the continuous berth allocation problem. Operations Research, 66(3):778-798, 2018.

[182] H.H. Yanasse and D.M. Katsurayama. Checkerboard pattern: proposals for its generation. International Transactions in Operational Research, 12(1):21-45, 2005.

[183] H.H. Yanasse and D.M. Katsurayama. An enumeration scheme to generate constrained exact checkerboard patterns. Computers $\&$ Operations Research, 35(6):2114-2128, 2008.

[184] G. Yu, Y. Mao, and J. Xiao. A new lower bound for online strip packing. European Journal of Operational Research, 250(3):754-759, 2016.

[185] G. Yu, Y. Mao, and J. Xiao. A new upper bound for the online square packing problem in a strip. Journal of Combinatorial Optimization, 33(4):1411-1420, 2017.

[186] G. Yu, Y. Mao, and J. Xiao. New upper bounds for online strip packing. Discrete Optimization, 23:20-32, 2017. 